\title{
Topological solitons make metamaterials crawl
}

\author{
Bolei Deng ${ }^{\mathrm{a}, *}$, Mohamed Zanaty ${ }^{\mathrm{a}, *}$, Antonio E. Forte ${ }^{\mathrm{a}, \mathrm{b}, \mathrm{c}}$, and Katia Bertoldi ${ }^{\mathrm{a}, \dagger}$ \\ a John. A. Paulson School of Engineering and Applied Sciences, Harvard University, Cambridge, MA 02138, USA; ${ }^{b}$ Department of Electronics, Information and Bioengineering, \\ Politecnico di Milano, Milan, 20133, Italy; ' Department of Engineering, King's College London, London, WC2R 2LS, UK
}

\begin{abstract}
In recent years, the ability to propagate topological solitons in mechanical metamaterials has unlocked unpaved paths towards potential applications in wave propagation, mechanical logic, and shape morphing. Here we demonstrate how a multistable metamaterial can harness topological solitons with coupled rotational and translational components, and become, itself, a crawling robot. We start by characterizing the topological solitons via experimental measurements and analysis. We then use their rotational component to produce a favorable gradient of friction between the metamaterial and the underlying substrate. This, in turn, creates locomotion. Previously proposed crawling robots have usually required complex control of multiple actuators. In contrast, our robot can be powered by a single actuator and all features needed for locomotion are embedded in the mechanics, and activated by the topological solitons.
\end{abstract}

Mechanical metamaterials, Multistability, Topological solitions, Locomotion, Crawling robots

\section{Introduction}

From snails and earthworms to maggots and amoebas, many organisms use waves in combination with friction control to locomote $(1,2)$. Their peculiar movements have inspired the design of robotic systems that harness elastic deformation to maneuver through confined spaces $(3,4)$. These robots typically comprise multiple actuators, which are precisely sequenced to achieve crawling (5-11). In an effort to reduce the complexity of the controls the most diverse phenomena have been harnessed, including mechanical instabilities (12$15)$, viscous forces $(16)$, solitory pulses $(17,18)$ and oscillating materials $(19,20)$. However, there still exist unraveled mechanisms that can be investigated and exploited towards this aim.

In recent years we have seen the rise of mechanical metamaterials as simple and effective platforms to control elastic waves $(21,22)$ in both the linear $(23-29)$ and the non-linear regimes (30-33). Through multistability, scientists have further expanded the capability of such metamaterials to support topological solitons (34-40): interfaces that occur to separate regions of different phases, also refereed to as domain walls (41-47), kinks $(34,48)$ and transition waves $(35-39,49)$. Not only such topological solitons display rich physics, but also have enabled a wide range of applications, including unidirectional propagation (35-39), energy harvesting (50-52), and mechanical logic (53).

Inspired by the rich physics of topological solitons, we here investigate how they can be exploited to make a multistable metamaterial crawl. We first focus on a one-dimensional (1D) chain of bistable unit cells - based on the rotating squares mechanism - and study its non-linear dynamic behavior. Such simple metamaterial platform has the advantage of supporting the propagation of topological solitons with two components that travel at the same speed - one translational and one rotational. Differently from the solitary pulses previously observed in the monostable mechanical metamaterial $(31,54)$, the topological solitons hereby investigated exhibit robustness to imperfections and do not require an impulsive trigger. As such, they represent an ideal platform for the design of simple machines that can be actuated with slow inputs and operate for many cycles. In particular, we demonstrate how such topological solitons mutate the $1 \mathrm{D}$ structure into a crawling device, for which the complexity of the controls is offloaded into the mechanics of the material itself. Moreover, we show that the proposed concept can be extended to $2 \mathrm{D}$ tessellations, opening avenues for simple and robust robotic structures capable of following arbitrarily complex trajectories.

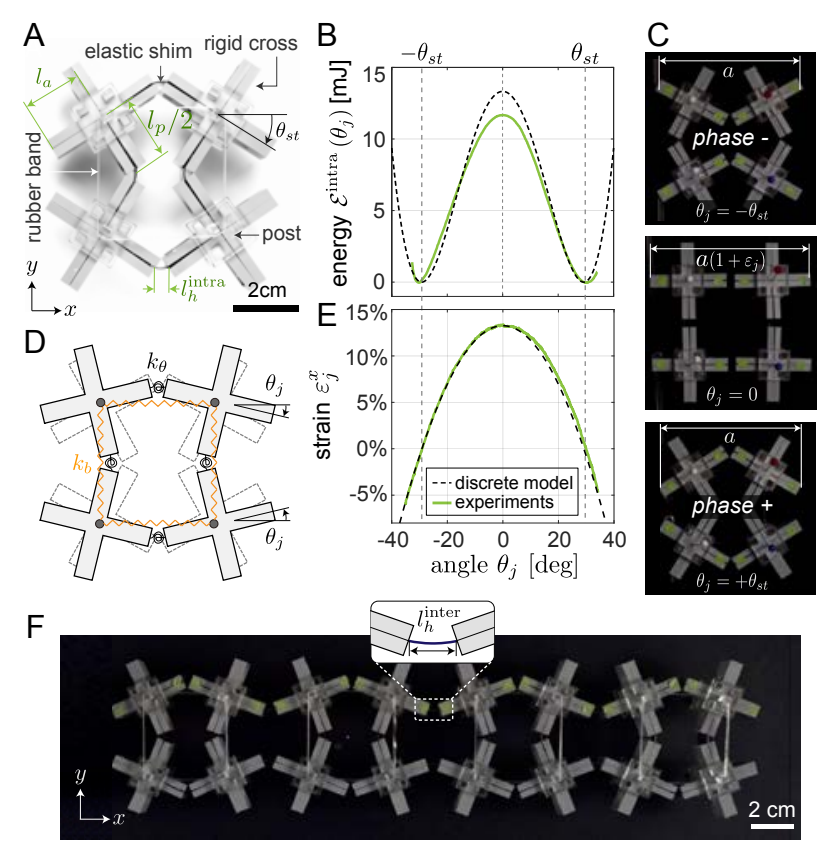

Fig. 1. Multistable mechanical metamaterial. (A) The bistable unit. (B) Elastic energy of the bistable unit, $\mathcal{E}^{\text {intra }}$, as predicted by experiments (solid line) and the discrete model (dashed line), (C) Snapshots of the bistable unit at its equilibrium $\left(\theta_{j}= \pm \theta_{s t}\right)$ and unstable $\left(\theta_{j}=0\right)$ states. (D) Schematics of the model. (E) Strain along the $x$-direction, $\epsilon_{j}^{x}$, as a function of the rotation of the crosses, $\theta_{j}$, for the bistable unit. Note that the rotation extracted from the experiments is the average of that of the top crosses. (F) A 1D chain comprising four bistable units connected by elastic hinges of length $l_{h}^{\text {inter }}$

*B.D. and M.Z. contributed equally to this work.

E-mail: bertoldi@seas.harvard.edu 


\section{Results}

Our System. The building block of our mechanical metamaterial is a bistable unit that comprises four rigid crosses connected by elastic hinges and a pre-stretched elastic band. The crosses have arm length $l_{a}=19 \mathrm{~mm}$, while the hinges have length $l_{h}^{\text {intra }}=3.5 \mathrm{~mm}$. A rubber band kept in place by posts rigidly connected to the crosses at a maximum distance $l_{p}=36 \mathrm{~mm}$ from each other (Fig. 1A) transforms the potential of the cell $\mathcal{E}^{\text {intra }}$ from convex to non-convex with two energy minima at $\theta_{j}=29.8^{\circ}$ and $-30.2^{\circ}$, where $\theta_{j}$ denotes the rotation of the top-right cross (Fig. 1B). These minima correspond to the two stable symmetric phases with all crosses rotated by the same amount but in alternating directions, which we refer to as phase+ and phase- (Fig. 1C). Note that $\mathcal{E}^{\text {intra }}$ was obtained by numerically integrating the reaction force of the unit measured experimentally, while accounting for the contribution of gravity (for more details about unit cell's geometry and experiments see Movie S1 and SI Appendix, section 1.1 and 2.2).

The bistable behavior of our building block can be captured by modelling the crosses as rigid bodies, the elastic hinges as rotational springs of stiffness $k_{\theta}=0.0103 \mathrm{~N} \cdot \mathrm{m}$ and the rubber band with four linear extension springs, each of stiffness $k_{b}=305 \mathrm{~N} / \mathrm{m}$ and rest length $l_{b}=27.5 \mathrm{~mm}$ (Fig. 1D). The elastic energy of the unit cell for such a discrete model takes the form

$$
\begin{aligned}
\mathcal{E}^{\text {intra }}\left(\theta_{j}\right) & =2 k_{b}\left(l_{p} \cos \theta_{j}-l_{b}\right)^{2}+8 k_{\theta} \theta_{j}^{2} \\
& \approx 2 k_{b}\left(l_{p}-l_{b}-l_{p} \theta_{j}^{2} / 2\right)^{2}+8 k_{\theta} \theta_{j}^{2}
\end{aligned}
$$

and is characterized by two minima corresponding to two stable phases and located at

$$
\theta_{j}= \pm \theta_{s t}= \pm \sqrt{2-\frac{2 l_{b}}{l_{p}}-\frac{8 k_{\theta}}{l_{p}^{2} k_{b}}}=29.4^{\circ}
$$

Such minima are separated by the energy barrier

$$
\Delta \mathcal{E}^{\text {intra }}=\mathcal{E}^{\text {intra }}(0)-\mathcal{E}^{\text {intra }}\left(\theta_{s t}\right)=\frac{1}{2} k_{b} l_{p}^{2} \theta_{s t}^{4}=13.8 \mathrm{~mJ} .
$$

As shown in Fig. 1B, $\mathcal{E}^{\text {intra }}$ matches the energy calculated from our experiments. Further, the model predicts a strain along the $x$-direction

$$
\varepsilon_{j}^{x}=\cos \theta_{j}-\cos \theta_{s t}
$$

and captures the temporary extension in $x$-direction observed in our experiments as the unit cell transitions from one stable state to the other (Fig. 1E, for details about the modeling of a unit cell see SI Appendix, section 3.1).

This bistable building block can form multistable metamaterials when arranged on a square grid. The single unit cells are connected through additional hinges of length $l_{h}^{\text {inter }} \in[3,15]$ $\mathrm{mm}$, creating a $1 \mathrm{D}$ chain or a $2 \mathrm{D}$ tessellation (Fig. $1 \mathrm{~F}$ and SI Appendix, section 1.2). In the following sections, we first investigate how the metamaterial supports the propagation of elastic non-linear pulses in 1D configurations and then study how such waves can be harnessed to make the system crawl, operated by minimal control.
Topological Solitons in 1D Chains. To investigate the propagation of non-linear pulses, we create a $1 \mathrm{D}$ chain of $1 \times N_{x}$ bistable units and excite one end using a linear actuator connected to the crosses of the leftmost unit via two levers. The levers convert the stroke of the actuator into rotation of the crosses and, therefore, force the leftmost unit to transition from one stable configuration to the other. As an example, in Figs. 2A-C we show a chain with five units connected by hinges of length $l_{h}^{\text {inter }}=6 \mathrm{~mm}$. As shown in Fig. $2 \mathrm{~A}$ all units are initially set in phase + . However, the transition to phase- slowly imposed at the leftmost unit by the actuator, triggers the propagation of a non-linear pulse that sequentially switches all units to phase-. Importantly, this pulse not only flips all $\theta_{j}\left(j=1, . ., N_{x}\right)$ sequentially from $\sim 28^{\circ}$ to $\sim-28^{\circ}$, but also temporarily displaces all units along the $x$-direction (Fig. 2B). Moreover, the results in Fig. 2C indicate that both the rotational and translational component of the pulse exhibit kink-like profiles, suggesting that the system supports the propagation of topological solitons that simultaneously activate two degrees of freedom (see SI Appendix, section 1.3 for more details on the actuation, sections 2.1 for the image processing procedure and Movie S2 for wave propagation examples of $1 \mathrm{D}$ chains with different $l_{h}^{\text {inter }}$ ).

Next, we investigate the effect of $l_{h}^{\text {inter }}$ and $N_{x}$ on the wave propagation. In Figs. 2D-F one can notice how the width of the pulses is highly affected by $l_{h}^{\text {inter }}$. In fact, short coupling hinges result in wide pulses, with all units deforming almost simultaneously. On the contrary, larger $l_{h}^{\text {inter }}$ values reduce the coupling between units and lead to narrower pulses. However, in the presence of dissipation due to friction between the metamaterial and the substrate this also limits the distance travelled by the waves. As an example, for $l_{h}^{\text {inter }}=8 \mathrm{~mm}$ the pulses fail to switch all units in a chain with $N_{x}=5$ cells, but propagates though the entire chain if $N_{x}=4$. Note that, in the presence of dissipation, for each $l_{h}^{\text {inter }}$ there exist a maximum number of unit cells able to propagate the pulse through its end (which we refer to as $N_{c}$ ). As shown in Fig. $2 \mathrm{G}$, $N_{c}$ monotonically decreases as the coupling hinges become longer and the propagating pulses get narrower. Similar trends are found for chains constructed using different unit cells characterized by $\theta_{s t}=25^{\circ}$ and $35^{\circ}$ (Figs. $2 \mathrm{H}$ and I).

To generalize and better understand the physics of the wave propagation in our system, we take advantage of a discrete model previously developed by the authors $(31,55)$. In the model, we assign to the $j^{\text {th }}$ bistable unit the elastic energy given by Eq. (1) and capture the behavior of the flexible coupling hinges using a combination of longitudinal and shearing springs with stiffness $k_{l}=72 \mathrm{~N} / \mathrm{mm}$ (31) and $k_{s}=\frac{1}{3} E w_{h}^{\text {inter }}\left(\delta_{h}^{\text {inter }} / l_{h}^{\text {inter }}\right)^{3}$, respectively. It follows that the elastic energy of the chain is given by

$$
\begin{aligned}
\mathcal{E}_{\text {ela }} & =\sum_{j=1}^{N_{x}} \mathcal{E}^{\text {intra }}\left(\theta_{j}\right)+\sum_{j=1}^{N_{x}-1}\left\{k_{s} l_{a}^{2}\left(\sin \theta_{j+1}-\sin \theta_{j}\right)^{2}\right. \\
& \left.+k_{l}\left[u_{j+1}-u_{j}+2 l_{a}\left(2 \cos \theta_{s t}-\cos \theta_{j+1}-\cos \theta_{j}\right)\right]^{2}\right\}
\end{aligned}
$$

and its Lagrangian can be written as

$$
\mathcal{L}=\sum_{j=1}^{N_{x}}\left[\frac{1}{2} m\left(\frac{\partial u_{j}}{\partial t}\right)^{2}+\frac{1}{2} J_{\theta}\left(\frac{\partial \theta_{j}}{\partial t}\right)^{2}\right]-\mathcal{E}^{\text {ela }}
$$



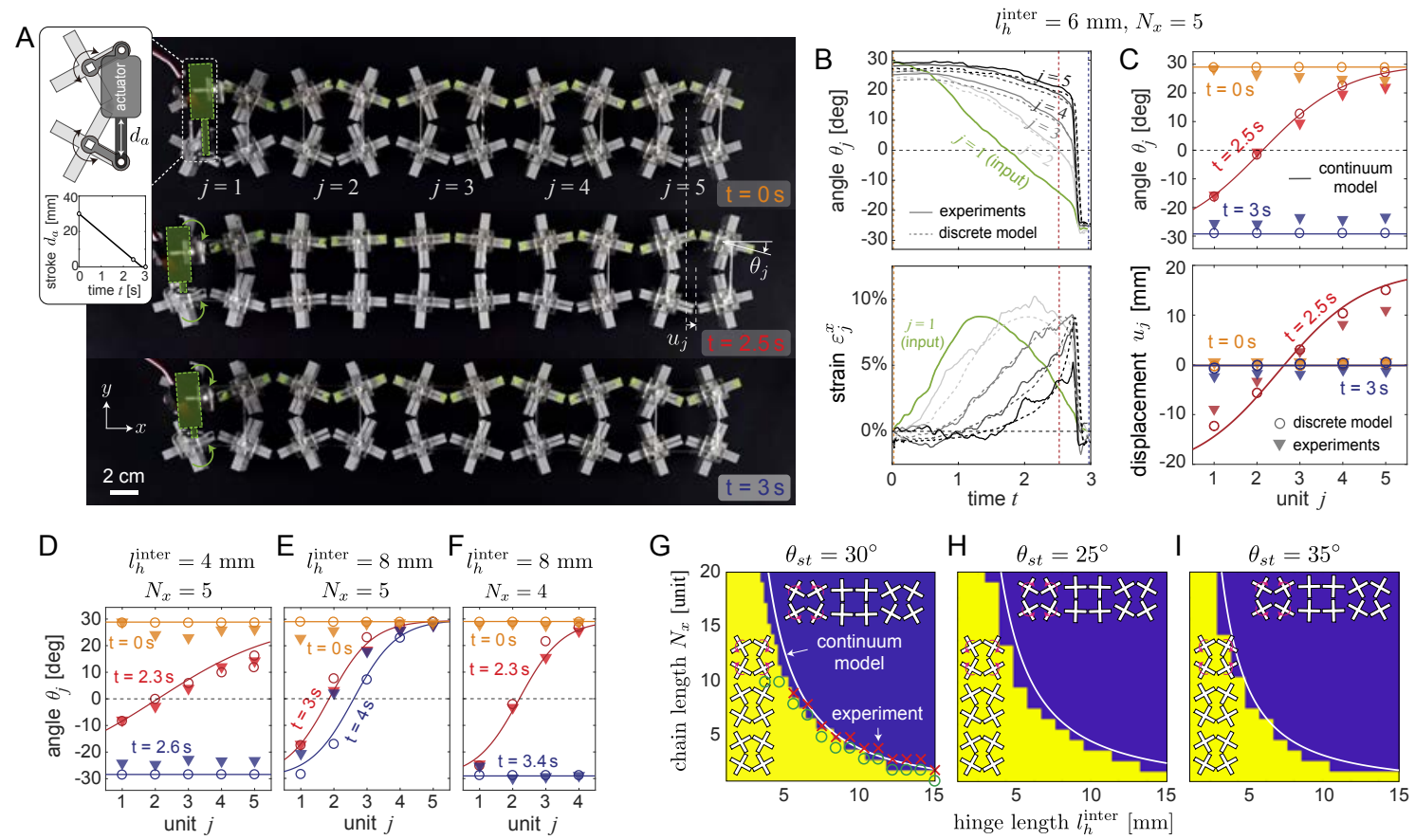

Fig. 2. Topological solitons in $1 \mathrm{D}$ chains. (A) Experimental snapshots of a metamaterial comprising $1 \times 5$ bistable units connected with hinges of length $l_{h}^{\text {inter }}=6$ mm at $t=0$ 2.5 and $3.0 \mathrm{~s}$. An actuator is mounted on the leftmost unit $(j=1$ ), whose stroke-time relation is shown in the inset. (B) Experimentally measured (solid lines) and numerically predicted (dashed lines) evolution of the rotation (top) and $x$-direction strain (bottom) of all bistable units as functions of time. (C) Rotation (top) and $x$-direction displacement (bottom) of every unit in the chain as a function of position at three different times as measured in experiments (triangular markers) and predicted by the discrete (circular markers) and continuum (solid lines). (D-F) Rotation of every unit for chains with (D) $l_{h}^{\text {inter }}=4 \mathrm{~mm}$ and $N_{x}=5$; (E) $l_{h}^{\text {inter }}=8 \mathrm{~mm}$ and $N_{x}=5 ;(\mathrm{F}) l_{h}^{\text {inter }}=8 \mathrm{~mm}$ and $N_{x}=4$. Note that the reported results for the continuum model are obtained by adjusting $\xi_{0}$ in Eq. (10) to best match the corresponding results for the discrete model. (G-I) Maps showing the combinations of $N_{x}$ and $l_{h}^{\text {inter }}$ resulting in pulses that reach the opposite end (yellow region for numerical results and green circular markers for experiments) or fail to switch all units (blue region for numerical results and red cross markers for experiments) in chains comprising units with $(\mathrm{G}) \theta_{s t}=30^{\circ},(\mathrm{H}) \theta_{s t}=25^{\circ}$ and $(\mathrm{I})$ $\theta_{s t}=35^{\circ}$. The white line corresponds to the prediction of Eq. (13).

where $t$ denotes time, $u_{j}$ is the displacement along the $x$ direction of the $j^{t h}$ unit, $m=45 \mathrm{~g}$ is the mass of the unit and $J_{\theta}=0.065 \mathrm{~g} \cdot \mathrm{m}^{2}$ is the inertia associated with $\theta_{j}$. To obtain the evolution of $\theta_{j}$ and $\varepsilon_{j}$ as functions of time, we then derive the equations of motion via the Euler-Lagrange equations and introduce Coulomb friction terms to account for the energy dissipation due to the sliding of the metamaterial on the surface (the friction coefficient of between the unit cells and the substrate is experimentally measured as $\mu=0.15$ see SI Appendix, section 3.3 for details). We then numerically integrate the equations of motion using the 4th order RungeKutta. When we apply the experimentally extracted input signal to the first unit and leave the opposite end free, our numerical analyses can successfully reproduce all the experimental results reported in Fig. 2 (see SI Appendix, section 3.2 for more information about the discrete model).

Once the model is validated we use it as a basis to derive an analytical solution for the system. To this end, we neglect dissipation and take the continuum limit of the discrete equations of motion to obtain

$$
\begin{aligned}
& m \frac{\partial^{2} u}{\partial t^{2}}=2 k_{l} \frac{\partial^{2} u}{\partial \xi^{2}}+8 k_{l} l_{a} \theta \frac{\partial \theta}{\partial \xi}, \\
& J_{\theta} \frac{\partial^{2} \theta}{\partial t^{2}}=2 k_{s} l_{a}^{2} \frac{\partial^{2}}{\partial \xi^{2}} \theta-\left(2 k_{b} l_{p}^{2}+8 k_{l} l_{a}^{2}\right)\left(\theta^{3}-\theta_{s t}^{2} \theta\right)-4 k_{l} l_{a} \theta \frac{\partial u}{\partial \xi}
\end{aligned}
$$

where $u(\xi, t)$ and $\theta(\xi, t)$ are continuous function that interpolate the displacement and rotation of the $j^{\text {th }}$ bistable unit as $u(\xi=j, t)=u_{j}(t)$ and $\theta(\xi=j, t)=\theta_{j}(t)$. By introducing the traveling coordinate $\zeta=\xi-c t$ (where $c$ denotes the wave velocity), integrating Eq. (7a) to obtain $\partial u / \partial \zeta$ and substituting that into Eq. (7b), we obtain

$$
\frac{\partial^{2}}{\partial \zeta^{2}} \theta=\frac{k_{b} l_{p}^{2}-\frac{4 k_{l} l_{a}^{2} c^{2} / c_{u}^{2}}{1-c^{2} / c_{u}^{2}}}{k_{s} l_{a}^{2}\left(1-c^{2} / c_{\theta}^{2}\right)} \theta\left(\theta-\theta_{s t}\right)\left(\theta+\theta_{s t}\right)
$$

where $c_{u}=\sqrt{2 k_{l} / m} \approx 1788 \mathrm{unit} / \mathrm{s}$ and $c_{\theta}=\sqrt{2 k_{s} l_{a}^{2} / J_{\theta}} \approx$ $311 \mathrm{unit} / \mathrm{s}$ are the characteristic velocities associated with linear longitudinal and rotational waves, respectively. Note that the pulse speed observed in our experiments $(c \approx 5$ unit/s) is much lower than $c_{u}$ and $c_{\theta}$, indicating that the system is over-damped and its behavior is not dominated by inertia effects. Therefore, we can assume that $c / c_{u} \rightarrow 0$ and $c / c_{\theta} \rightarrow 0$ and Eq. (8) simplifies to

$$
\frac{\partial^{2}}{\partial \zeta^{2}} \theta=\frac{k_{b} l_{p}^{2}}{k_{s} l_{a}^{2}} \theta\left(\theta-\theta_{s t}\right)\left(\theta+\theta_{s t}\right) .
$$

Eq. (9) is a Klein-Gordon equation with quadratic and cubic nonlinearities, which can be also derived from the well-known $\phi^{4}$ field equation describing phase transitions in solid-state materials $(56,57)$. By solving Eq. (9), substituting the solution into Eq. (7a) and integrating the latter with respect to $\zeta$, we 
obtain

$$
\left[\begin{array}{l}
\theta \\
u
\end{array}\right]=\left[\begin{array}{c} 
\pm \theta_{s t} \\
-2 l_{a} W \theta_{s t}^{2}
\end{array}\right] \tanh \left(\frac{\xi-\xi_{0}}{W}\right),
$$

where $\xi_{0}$ is a position shift, and

$$
W=\frac{l_{a}}{\theta_{s t} l_{p}} \sqrt{\frac{2 k_{s}}{k_{b}}}=\sqrt{\frac{k_{s} l_{a}^{2} \theta_{s t}^{2}}{\Delta \mathcal{E}^{\text {intra }}}}
$$

denotes the width of the pulse, which is determined by the ratio between the coupling energy $\left(\sim k_{s} l_{a}^{2} \theta_{s t}^{2}\right)$ and the local energy barrier $\left(\Delta \mathcal{E}^{\text {intra }}\right)$. Importantly, Eq. (10) confirms that the pulses observed in our system are topological solitons with a rotational and translational components that co-propagate through the system with identical width and velocity. It is important to note that such topological solitons are fundamentally different from the vector solitons recently reported in monostable metamaterials based on the rotating square mechanism $(30,31)$, as the geometrical incompatibility between phase+ and phase- make them robust, so that they can be initiated even by very slow inputs - a feature that will be use to realize a simple crawler robot. As shown in Figs. 2C-F, the derived analytical solution matches well all experimental and numerical results and captures the effect of $l_{h}^{\text {inter }}$ on the profile of the topological solitons. Therefore, since the shape of the pulses observed in our experiments can be nicely captured by the analytical solution even when they fail to propagate through the entire chain (despite the fact that the model does not account for friction), we can use the model to predict $N_{c}$. Towards this end, we first estimate the energy of a pulse supported by the system, $\mathcal{E}^{\text {ts }}$, by taking the continuum limit of Eq. (5) and subtracting the energy of its initial configuration

$$
\mathcal{E}_{\mathrm{ts}} \approx 2 \theta_{s t}^{3} l_{a} l_{p} \sqrt{2 k_{s} k_{b}} .
$$

We then calculate $N_{c}$ by comparing $\mathcal{E}_{\text {ts }}$ to the energy required to switch a unit cell from one stable state to the other

$$
N_{c}=\frac{\mathcal{E}_{\mathrm{ts}}}{\Delta \mathcal{E}^{\text {intra }}}=\frac{4 l_{a}}{\theta_{s t} l_{p}} \sqrt{\frac{2 k_{s}}{k_{b}}}=4 W,
$$

and find that it matches both numerical and experimental results (see solid line in Fig. 2 G-I and for additional information about the analytical model please see SI Appendix, section $3.5)$.

\section{Locomotion via Topological Solitons. After demonstrating} that our system supports the propagation of topological solitons with coupled rotational and translational components, we now move into investigating how to harness these components to make the metamaterial crawl with minimal control.

It is known that limbless locomotion requires propagation of contraction/extension waves combined with the controlled formation of anchors to provide increased local sliding resistance $(2,58)$. Therefore we aim at exploiting the rotational component of the topological soliton to generate dynamic anchoring points and the translational one to generate extension/contraction. To realize this, we add a pair of wheels to each cross in the chain, which are oriented along the $x$ direction for $\theta_{j}=0$ (Fig. 3A). The interaction between the wheels and the substrate generates a resistive force along the $x$-direction and torque applied to the $j^{\text {th }}$ unit

$$
\begin{aligned}
F_{j}^{\text {fric }, x} & =-m g \mu_{s}\left|\sin \theta_{j}\right| \operatorname{sign}\left[\frac{\partial u_{j}}{\partial t}\right], \\
M_{j}^{\text {fric }} & =-m g \mu_{s} d_{\mathrm{wh}} \operatorname{sign}\left[\frac{\partial \theta_{j}}{\partial t}\right],
\end{aligned}
$$

where $g$ is the is the gravitational constant and $d_{\mathrm{wh}}=20 \mathrm{~mm}$ is the distance between the two wheels under the same cross, and $\mu_{s}$ is the friction coefficient with the substrate. Eq. (14) clearly shows that, while $M_{j}^{\text {fric }}$ is constant, $F_{j}^{\text {fric, } x}$ is highly affected by $\theta_{j}$ (Fig. 3B): it vanishes for $\theta_{j}=0$ (when the wheels are purely rolling) and monotonically increases as the crosses rotate and the units approach their stable states at $\theta_{j}= \pm \theta_{s t}$ (Movie S3). As such, we expect the bistable units to be able to elongate and slide when transitioning between the two stable states and to provide anchor points when in their stable configurations.

The sequential switching of the units induced by the pulses is key in the locomotion process: certain portions of the array extend and slide, while others provide resistance against backsliding. In Fig. $3 \mathrm{C}$ we report experimental snapshots of a wheeled chain with $N_{x}=3$ and $l_{h}^{\text {inter }}=6 \mathrm{~mm}$. One can notice that when the first unit transitions from phase- to phase+, the second and the third ones are still in phase- and therefore experience large frictional forces. As such, they act as anchor points and force the first unit to expand leftwards. Next, when the first unit reaches phase+, it becomes an anchoring point and prevents backsliding as the two other units transition between states. As a result, after the topological soliton switches all units to phase+, the structure has moved towards the left. Finally, when the actuator brings the first unit back to the initial state, the process repeats and at the end of the full cycle the chain has shifted by $d_{\text {step }}=14.5 \mathrm{~mm}$ towards the left (Movie S4). Note that the distance travelled by the system during the two half cycles is not identical as unavoidable imperfections during fabrication lead to an asymmetric energy profile with two minima of slightly different heights (Fig. S22).

Furthermore, we expand our discrete model in order to capture the rich behaviour of the structure during locomotion and efficiently explore the design space. In particular, we account for friction to model the interaction of the structure with the underlying surface and, therefore capture its ability to crawl. As shown in Fig. 3D, the numerical simulations are in good agreement with the experimental results. Having confirmed the validity of our numerical model, we next use it to study the effect of $N_{x}$ and $l_{h}^{\text {inter }}$ on the locomotion of the wheeled chains. Both experiments and simulations indicate that there is an optimum value of $l_{h}^{\text {inter }}$ for which the chain moves more efficiently (Fig. 3E). Shorter hinges result in wider pulses and therefore in a lack of phase difference between units. Differently, larger $l_{h}^{\text {inter }}$ lead to sharp pulses that minimally extend the chain. Further, it is worth noticing that for $l_{h}^{\text {inter }}<2 \mathrm{~mm}$ all units transition between phases almost simultaneously, hindering locomotion. The same occurs for $l_{h}^{\text {inter }}>10 \mathrm{~mm}$ where the topological solitons do not have enough energy to propagate through the entire chain (for details about the discrete modeling of locomotion, see SI Appendix, section 3.2). When using our model to scan the entire design space, we conclude that locomotion is only achieved if $N_{x}$ and $l_{h}^{\text {inter }}$ are chosen so that the pulses are able to propagate through the entire chain (i.e. $N_{x}<N_{c}$ ) and meanwhile their width is smaller that the length of the chain (i.e. $N_{x}>W$ ). This is clearly 

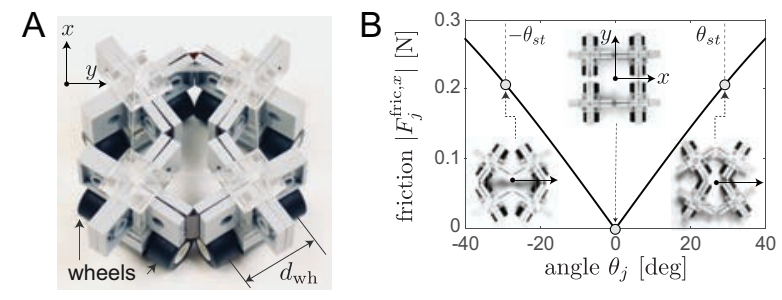

C
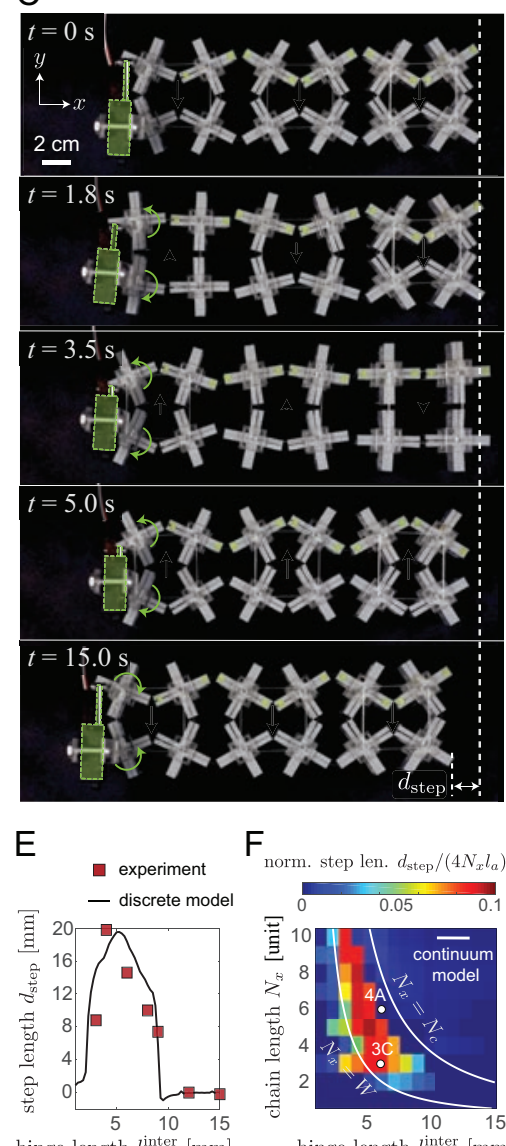

$\mathrm{F}$

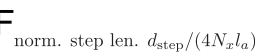

D experiment: $\nabla \theta_{j} \nabla u_{j}$

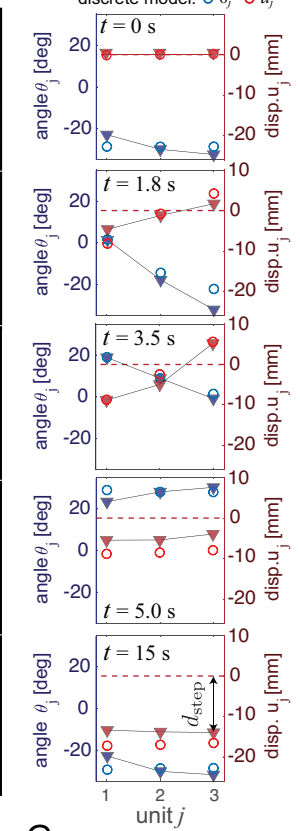

G
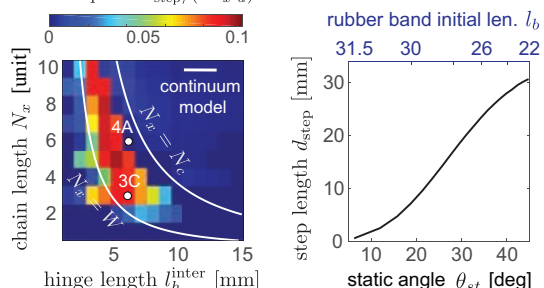

hinge length $l_{h}^{\text {inter }}[\mathrm{mm}]$ hinge length $l_{h}^{\text {inter }}[\mathrm{mm}]$ $l_{b}$ ) have also a large impact on the step length. Specifically, our numerical analyses on a $N_{x}=3$ and $l_{h}^{\text {inter }}=6 \mathrm{~mm}$ chain indicate that $d_{\text {step }}$ monotonically increase with $\theta_{s t}$, as larger $\theta_{s t}$ increases the temporary extension of the units associated to their phase transition (Fig. 3G, Fig. S20 and Movie S4). Further, our model indicates that, when the pulses are able to propagate through the entire chain, the robot crawls with an efficiency of $\sim 3 \%$ (Fig. S19). Although low, such efficiency is comparable to that typically reported for the locomotion of soft robots (59).

Finally, in all our experiments the actuator takes $5 \mathrm{~s}$ to switch the carrying unit and, therefore, initiate the topological solitons. By shortening the actuation time the crawling speed of the robot can be increased. However, such actuation time cannot be decreased indefinitely, since eventually it will approach the time that the pulse takes to propagate through the chain. This is the limit condition at which the topological solitons are unable to reach the end of the structure before the opposite phase is triggered by the actuator, hindering the locomotion mechanism (see Fig. S21 and Movie S4).

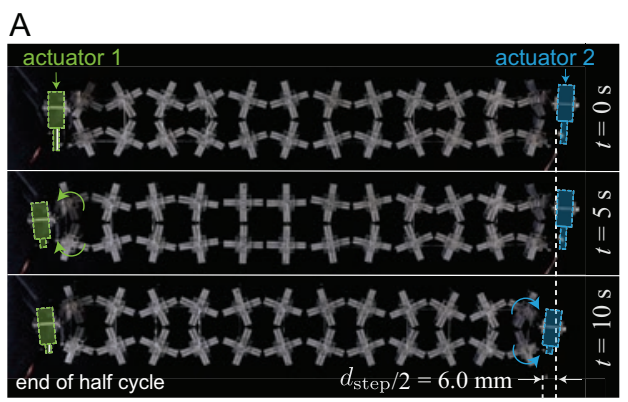

B
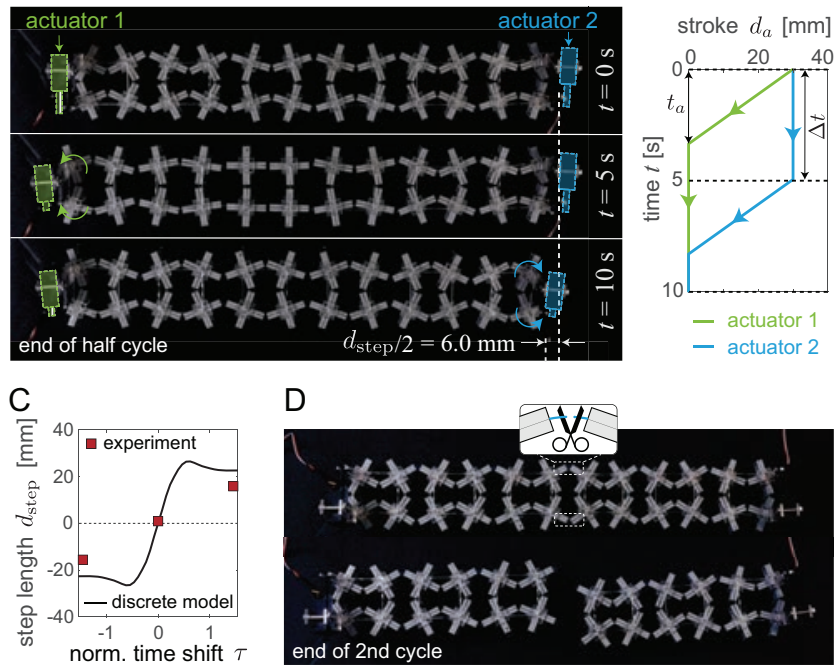

Fig. 4. Wheeled chain with two actuators. (A) Experimental snapshots of a wheeled chain with $N_{x}=6, l_{h}^{\text {inter }}=6 \mathrm{~mm}$ and two actuators connected to the leftmost and rightmost units at $t=0,5$ and $10 \mathrm{~s}$. (B) The stroke of the two actuators as a function of $t, t_{a}$ corresponds to the actuation time and $\tau t$ to the difference between the times at which the two actuators are activated. (C) Evolution of the step length $d_{\text {step }}$ as a function of the normalized time shift $\tau$ as measured in experiments (markers) and predicted by the discrete model (solid line). (D) When the hinges connecting units $j=3$ and $j=4$ fail, the chain splits into two independent and functional systems.

Our design space can be further expanded if multiple actuators are used to manipulate the pulses. As an example, in Fig. 4A we show a chain that is unable to propagate a topological soliton through its end, as the previous results demonstrate $\left(N_{x}=6\right.$ and $\left.l_{h}^{\text {inter }}=6 \mathrm{~mm}\right)$. To make this chain crawl, we connect two actuators to the leftmost and rightmost units (which we refer to as actuator 1 and actuator 2, respectively). We start by contracting actuator 1 to switch the leftmost unit cell in $3.5 \mathrm{~s}$ (Fig. 4B). This initiates a pulse that propagates rightwards, but stops at the center of the chain as the rightmost unit cannot switch because of actuator 2 . However, by activating actuator 2 at $t=5 \mathrm{~s}$, we can guide the 
A
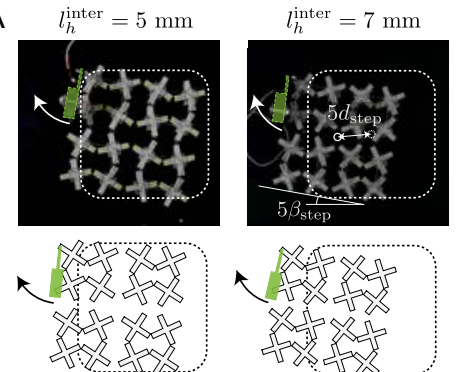

(after 5 cycles)

C
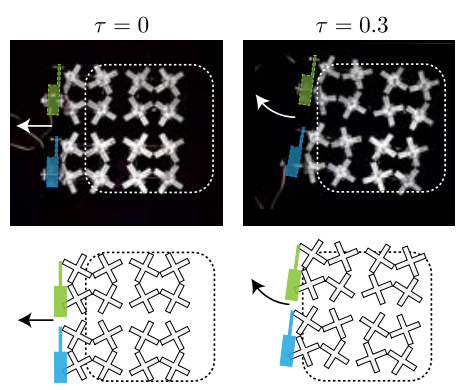

$\left(l^{\text {inter }}=11 \mathrm{~mm}\right.$, after 5 cycles $)$
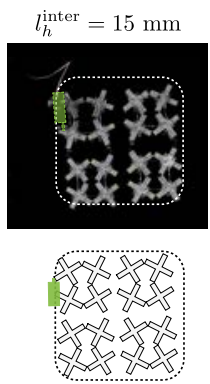

$\tau=-0.3$
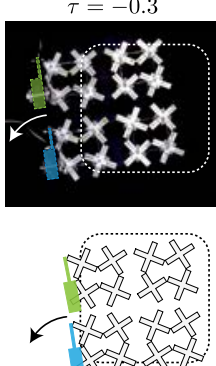

B

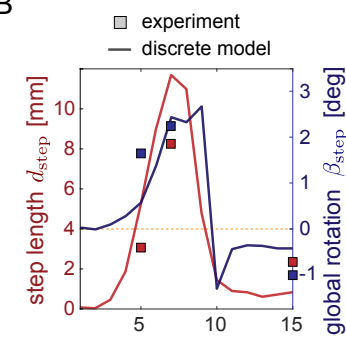

hinge length $l_{h}^{\text {inter }}[\mathrm{mm}]$

D

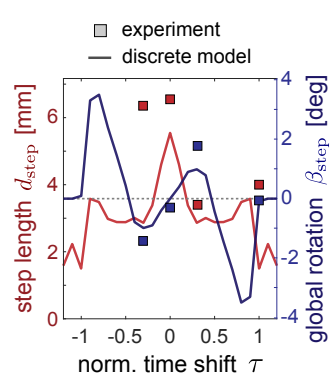

$\mathrm{E}$
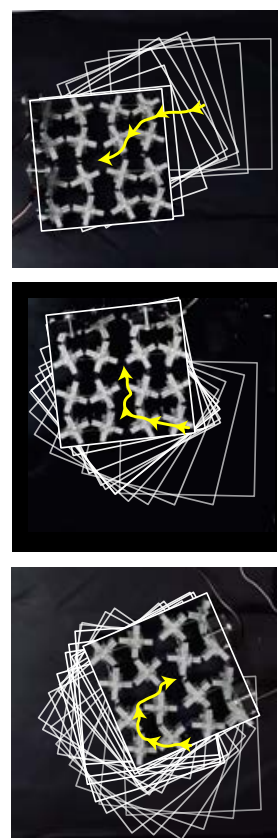

Fig. 5. Locomotion of 2D structures. (A) Experimental (top) and numerical snapshots showing a $2 \times 2$ structure with $l_{h}^{\text {inter }}=5,7,15 \mathrm{~mm}$ and one actuator connected to the top-left unit after 5 cycles of actuation. The dashed squares indicate their initial positions. (B) Effect of $l_{h}^{\text {inter }}$ on the step length $d_{\text {step }}$ and global rotation $\beta_{\text {step }}$ for a $2 \times 2$ metamaterial with an actuator connected to the top-left unit. (C) Experimental (top) and numerical snapshots (bottom) showing a $2 \times 2$ structure with $l_{h}^{\text {inter }}=11$ mm and two actuators connected to the left units after 5 cycles of actuation for different normalized time shift $\tau=0,0.3$ and -0.3 . The dashed squares indicate their initial positions. (D) Effect of $\tau$ on $d_{\text {step }}$ and $\beta_{\text {step }}$ for a $2 \times 2$ metamaterial with $l_{h}^{\text {inter }}=11 \mathrm{~mm}$ and actuators connected to both units on the left. (E) By varying $\tau$ we can guide the structure along complex paths. The dashed squares indicate their intermediate configurations and the yellow arrows represent their trajectories reached upon actuation. Note that we only present the final configurations of the structure and use dashed lines to indicate its intermediate positions. Since the metamaterial return to its initial phase after each cycle, the final configuration looks the same as the initial one except for a rigid body shift.

trapped pulse to propagate towards the right end, switching all the remaining units and making the structure move by $d_{\text {step }} / 2=6 \mathrm{~mm}$ leftwards at $t=10 \mathrm{~s}$.

Further control on locomotion can be achieved by tuning the normalized time shift between the two actuators, $\tau$. This is defined as the ratio between the difference in time at which the actuators are activated, $\Delta t$, and the time of actuation, $t_{a}$. As shown in Fig. 4C, if the two actuators move simultaneously (i.e., $\tau=0 \mathrm{~s}$ ), all the units switch between their stable states at the same time, no wave propagates and no locomotion occurs. Differently, if actuator 1 is activated before actuator 2 (i.e., $\tau>0$ ), the chain moves leftwards, whereas, if actuator 2 is activated first (i.e., $\tau<0$ ), the system moves rightwards. Finally, the second actuator not only enables us to control the direction of motion, but also improves the robustness of the system, as in case of a unit failure (i.e. a pair of shims are cut) the chain splits into two independent and functional systems (Fig. 4D). For an animated demonstration of the above experiments see Movie S5.

Locomotion of 2D Structures. In order to expand the range of motions, we focus on 2D tessellations, as these have been recently shown to support propagation of transition waves along complex paths (38) (see Movie S6 for evidence of the propagation of waves in our $2 \mathrm{D}$ system). Hence, we consider a structure comprising $2 \times 2$ bistable units and an actuator connected to its top-left cell. As shown in Fig. 5A, we find that depending on the length of such hinges, the structure not only translates but also rotates. For example, for $l_{h}^{\text {inter }}=5$ $\mathrm{mm}$ the structure translates and rotates, for $l_{h}^{\text {inter }}=7 \mathrm{~mm}$ both the rotation and translation are further accentuated, whereas for $l_{h}^{\text {inter }}=15 \mathrm{~mm}$ the structure moves only minimally. If we define $d_{\text {step }}$ as the distance travelled by the center of the structure, and $\beta_{\text {step }}$ the angle of rotation in the $x-y$ plane, our numerical results indicate that these two measures are strongly correlated and that their dependence on $l_{h}^{\text {inter }}$ is similar to that observed for $d_{\text {step }}$ in a $1 \mathrm{D}$ chain (Fig. 5B).

When increasing the number of actuators to two it is possible to achieve more control on the locomotion. For example, if one places two actuators to both left units of a $2 \times 2$ structure with $l_{h}^{\text {inter }}=11 \mathrm{~mm}$, it is possible to direct the structure to navigate straight, rotate clockwise and counterclockwise. The different modes of locomotion can be chosen by selecting the time at which the two actuators are activated, i.e. their normalized time shift $\tau$ (Fig. 5C). Further, our numerical results indicate a highly non-linear and complex dependence of both $\beta_{\text {step }}$ and $d_{\text {step }}$ from $\tau$ (Fig. 5D). As such, by simply varying $\tau$ we can guide the structure along complex paths comprising straight segments and turns (Fig. 5E and Movie S7).

\section{Conclusion}

To summarize, we have shown that the propagation of topological solitons with coupled translational and rotational components in multistable metamaterials based on the square 
rotating mechanism can be harnessed to achieve locomotion Specifically, we have exploited the translational component of the solitons to generate sequential extensions/contractions and the rotational component to generate dynamic anchoring points with the help of wheels. Further, we have developed an analytical model that not only fully describes the characteristics of the supported topological solitons, but also provides guidelines for the selection of geometrical parameters resulting in locomotion. We have initially focused on a 1D chain, but then demonstrated that the concept can be extended to $2 \mathrm{D}$ structures and that by introducing multiple actuators to initiate the pulses we can control the direction of motion as well as make turns. Unlike previously proposed crawling robots that require complex input control or material processing $(5-11,19,20)$, our system is easy to build and requires a simple and slow input mechanical signal to operate. This is because the proposed metamaterial intrinsically possesses all functionalities that are essential for locomotion (i.e. the ability to sequentially extend/contract and to create dynamic anchor points). The propagation of topological solitons activates them and provides the sequence required to achieve locomotion, significantly simplifying the input control. Note that the topological nature of the pulses plays a crucial role, as no locomotion is observed when the unit cells are monostable (see Fig. S9). Further, owing to the robustness of the topological solitons, our locomotion strategy is found to work also in the presence of imperfections, including non-symmetric potential wells (see Fig. S23).

While in this study we have considered units equipped with wheels to move on a solid substrate, the concept can be extended to different environments. For example, a skating robot can be realized by attaching blades to the units, while a swimmer can be obtained by introducing paddles. Moreover advanced functionality can be achieved by considering larger tessellations of different shapes, by manufacturing the unit cells to micrometer scale, and by introducing control algorithms based on neural network or unsupervised learning (60). As such, we believe that the proposed metamaterial has potential to serve as a platform for the next generation of crawling robots.

Methods. Details of fabrication, assembly and actuation are provided in SI Appendix, Section S1. Details on testing are provided in SI Appendix, Section S2. Details of the discrete and continuum mathematical model are provided in SI Appendix, Section S3.

Data Availability. The experimental and numerical data in support of the findings in this study are available from the corresponding author upon request.

Code Availability. Matlab code for discrete simulations can be found at: https://github.com/boleideng94/topological_locomotion

Acknowledgments. Research was supported by the NSF grants DMR-2011754, DMR-1922321 and EFRI-1741685. M.Z. acknowledges the financial support provided by the Swiss National Science Foundation under grant number P2ELP2-184497. A.E.F. acknowledges that this project has received funding from the European Union's Horizon 2020 research and innovation programme under the Marie Skłodowska-Curie grant agreement No 798244. We thank Anqi Chen for assistance on the friction measurements. We thank Connor McCann for input on the manuscript.

Author Contributions. B.D, M.Z., A.E.F. and K.B. conceived the project; B.D and M.Z designed the metamaterial; M.Z. designed and performed the experiments; B.D. developed the mathematical model and conducted simulations; B.D., M.Z., A.E.F and K.B. analyzed the data and wrote the paper.

Competing Interests. The authors declare no competing interests.

\section{References}

1. Dirk Dormann and Cornelis J Weijer. Propagating chemoattractant waves coordinate periodic cell movement in dictyostelium slugs. Development, 128(22):4535-4543, 2001.

2. Shigeru Kuroda, Itsuki Kunita, Yoshimi Tanaka, Akio Ishiguro, Ryo Kobayashi, and Toshiyuk Nakagaki. Common mechanics of mode switching in locomotion of limbless and legged animals. Journal of the Royal Society interface, 11(95):20140205, 2014.

3. Sangbae Kim, Cecilia Laschi, and Barry Trimmer. Soft robotics: a bioinspired evolution in robotics. Trends in biotechnology, 31(5):287-294, 2013.

4. Deepak Trivedi, Christopher D Rahn, William M Kier, and lan D Walker. Soft robotics: Biolog ical inspiration, state of the art, and future research. Applied bionics and biomechanics, 5(3): 99-117, 2008.

5. Masahiro Shimizu, Akio Ishiguro, and Toshihiro Kawakatsu. Slimebot: A modular robot that exploits emergent phenomena. In Proceedings of the 2005 IEEE International Conference on Robotics and Automation, pages 2982-2987. IEEE, 2005.

6. Michael Rubenstein, Ying Sai, Cheng-Ming Chuong, and Wei-Min Shen. Regenerative patterning in swarm robots: mutual benefits of research in robotics and stem cell biology. The International journal of developmental biology, 53(5-6):869, 2009.

7. Daniela Rus and Marsette Vona. Crystalline robots: Self-reconfiguration with compressible unit modules. Autonomous Robots, 10(1):107-124, 2001.

8. Victor Zykov, Efstathios Mytilinaios, Bryant Adams, and Hod Lipson. Self-reproducing machines. Nature, 435(7039):163-164, 2005

9. Jianing Chen, Melvin Gauci, Wei Li, Andreas Kolling, and Roderich Groß. Occlusionbased cooperative transport with a swarm of miniature mobile robots. IEEE Transaction on Robotics, 31(2):307-321, 2015.

10. Shuguang Li, Richa Batra, David Brown, Hyun-Dong Chang, Nikhil Ranganathan, Chuck Hoberman, Daniela Rus, and Hod Lipson. Particle robotics based on statistical mechanics of loosely coupled components. Nature, 567(7748):361-365, 2019

11. William Savoie, Thomas A Berrueta, Zachary Jackson, Ana Pervan, Ross Warkentin, Shengkai Li, Todd D Murphey, Kurt Wiesenfeld, and Daniel I Goldman. A robot made of robots: Emergent transport and control of a smarticle ensemble. Science Robotics, 4(34): eaax4316, 2019.

12. Ahmad Rafsanjani, Yuerou Zhang, Bangyuan Liu, Shmuel M Rubinstein, and Katia Bertold Kirigami skins make a simple soft actuator crawl. Science Robotics, 3(15):eaar7555, 2018.

13. Daniel J Preston, Haihui Joy Jiang, Vanessa Sanchez, Philipp Rothemund, Jeff Rawson, Markus P Nemitz, Won-Kyu Lee, Zhigang Suo, Conor J Walsh, and George M Whitesides. A soft ring oscillator. Science Robotics, 4(31):eaaw5496, 2019.

14. Tian Chen, Osama R Bilal, Kristina Shea, and Chiara Daraio. Harnessing bistability for directional propulsion of soft, untethered robots. Proceedings of the National Academy of Sciences, 115(22):5698-5702, 2018.

15. Larissa S Novelino, Qiji Ze, Shuai Wu, Glaucio H Paulino, and Ruike Zhao. Untethered control of functional origami microrobots with distributed actuation. Proceedings of the National Academy of Sciences, 117(39):24096-24101, 2020

16. Nikolaos Vasios, Andrew J Gross, Scott Soifer, Johannes TB Overvelde, and Katia Bertold Harnessing viscous flow to simplify the actuation of fluidic soft robots. Soft Robotics, 7(1): $1-9,2020$.

17. Bolei Deng, Liyuan Chen, Donglai Wei, Vincent Tournat, and Katia Bertoldi. Pulse-driven robot: Motion via solitary waves. Science Advances, 6(18):eaaz1166, 2020.

18. Nikolai Gorbushin and Lev Truskinovsky. Peristalsis by pulses of activity. arXiv preprint arXiv:2012.07017, 2020.

19. Anne Helene Gelebart, Dirk Jan Mulder, Michael Varga, Andrew Konya, Ghislaine Vantomme, EW Meijer, Robin LB Selinger, and Dirk J Broer. Making waves in a photoactive polymer film. Nature, 546(7660):632-636, 2017.

20. Hongri Gu, Quentin Boehler, Haoyang Cui, Eleonora Secchi, Giovanni Savorana, Carmela De Marco, Simone Gervasoni, Quentin Peyron, Tian-Yun Huang, Salvador Pane, et al. Magnetic cilia carpets with programmable metachronal waves. Nature communications, 11(1) $1-10,2020$.

21. Mahmoud I Hussein, Michael J Leamy, and Massimo Ruzzene. Dynamics of phononic materials and structures: Historical origins, recent progress, and future outlook. Applied Mechanics Reviews, 66(4), 2014

22. Johan Christensen, Muamer Kadic, Oliver Kraft, and Martin Wegener. Vibrant times for mechanical metamaterials. Mrs Communications, 5(3):453-462, 2015.

23. Pai Wang, Filippo Casadei, Sicong Shan, James C Weaver, and Katia Bertoldi. Harnessing buckling to design tunable locally resonant acoustic metamaterials. Physical review letters, 113(1):014301, 2014

24. Shu Zhang, Chunguang Xia, and Nicholas Fang. Broadband acoustic cloak for ultrasound waves. Physical review letters, 106(2):024301, 2011.

25. Pai Wang, Ling Lu, and Katia Bertoldi. Topological phononic crystals with one-way elastic edge waves. Physical review letters, 115(10):104302, 2015.

26. Zhengyou Liu, Xixiang Zhang, Yiwei Mao, YY Zhu, Zhiyu Yang, Che Ting Chan, and Ping Sheng. Locally resonant sonic materials. science, 289(5485):1734-1736, 2000.

27. Marc Serra-Garcia, Valerio Peri, Roman Süsstrunk, Osama R Bilal, Tom Larsen, Luis Guillermo Villanueva, and Sebastian D Huber. Observation of a phononic quadrupole topological insulator. Nature, 555(7696):342-345, 2018.

28. Roman Süsstrunk and Sebastian D Huber. Observation of phononic helical edge states in a mechanical topological insulator. Science, 349(6243):47-50, 2015.

29. Lisa M Nash, Dustin Kleckner, Alismari Read, Vincenzo Vitelli, Ari M Turner, and William TM Irvine. Topological mechanics of gyroscopic metamaterials. Proceedings of the National Academy of Sciences, 112(47):14495-14500, 2015.
449 
Bolei Deng, Jordan Raney, Vincent Tournat, and Katia Bertoldi. Elastic vector solitons in soft architected materials. Physical Review Letters, 118(20), 2017.

31. Bolei Deng, Pai Wang, Qi He, Vincent Tournat, and Katia Bertoldi. Metamaterials with amplitude gaps for elastic solitons. Nature Communications, 9:3410, 2018. . URL https: //doi.org/10.1038/s41467-018-05908-9.

32. Hiromi Yasuda, Yasuhiro Miyazawa, Efstathios G Charalampidis, Christopher Chong, Panayotis $\mathrm{G}$ Kevrekidis, and Jinkyu Yang. Origami-based impact mitigation via rarefaction solitary wave creation. Science advances, 5(5):eaau2835, 2019.

33. Chengyang Mo, Jaspreet Singh, Jordan R Raney, and Prashant K Purohit. Cnoidal wave propagation in an elastic metamaterial. Physical Review E, 100(1):013001, 2019.

34. Yafei Zhang, Bo Li, QS Zheng, Guy M Genin, and CQ Chen. Programmable and robust static topological solitons in mechanical metamaterials. Nature communications, 10(1):1-8, 2019.

35. Neel Nadkarni, Andres F Arrieta, Christopher Chong, Dennis M Kochmann, and Chiara Daraio. Unidirectional transition waves in bistable lattices. Physical review letters, 116(24): 244501, 2016.

36. Yisheng Zheng, Zhen Wu, Xinong Zhang, and KW Wang. A piezo-metastructure with bistable circuit shunts for adaptive nonreciprocal wave transmission. Smart Materials and Structures, 28(4):045005, 2019

37. Hongbin Fang, KW Wang, and Suyi Li. Asymmetric energy barrier and mechanical diode effect from folding multi-stable stacked-origami. Extreme Mechanics Letters, 17:7-15, 2017.

38. Lishuai Jin, Romik Khajehtourian, Jochen Mueller, Ahmad Rafsanjani, Vincent Tournat, Katia Bertoldi, and Dennis M Kochmann. Guided transition waves in multistable mechanical metamaterials. Proceedings of the National Academy of Sciences, 117(5):2319-2325, 2020.

39. H Yasuda, LM Korpas, and JR Raney. Transition waves and formation of domain walls in multistable mechanical metamaterials. Physical Review Applied, 13(5):054067, 2020.

40. Dian Yang, Lihua Jin, Ramses V Martinez, Katia Bertoldi, George M Whitesides, and Zhigang Suo. Phase-transforming and switchable metamaterials. Extreme Mechanics Letters, 6:1-9, 2016

1. Walter J Merz. Domain formation and domain wall motions in ferroelectric bati o 3 single crystals. Physical Review, 95(3):690, 1954.

42. M Yamanouchi, D Chiba, F Matsukura, and $\mathrm{H}$ Ohno. Current-induced domain-wall switching in a ferromagnetic semiconductor structure. Nature, 428(6982):539-542, 2004.

43. Jordan E Massad and Ralph C Smith. A domain wall model for hysteresis in ferroelastic materials. Journal of Intelligent Material Systems and Structures, 14(7):455-471, 2003.

44. DI Paul, J Marquiss, and D Quattrochi. Theory of magnetization: Twin boundary interaction in ferromagnetic shape memory alloys. Journal of applied physics, 93(8):4561-4565, 2003.

45. Dan A Allwood, Gang Xiong, CC Faulkner, D Atkinson, D Petit, and RP Cowburn. Magnetic domain-wall logic. Science, 309(5741):1688-1692, 2005.

46. JR Barkley, LH Brixner, EM Hogan, and RK Waring Jr. Control and application of domain wall motion in gadolinium molybdate. Ferroelectrics, 3(1):191-197, 1972.

7. Stuart SP Parkin, Masamitsu Hayashi, and Luc Thomas. Magnetic domain-wall racetrack memory. Science, 320(5873):190-194, 2008

48. Thierry Dauxois and Michel Peyrard. Physics of solitons. Cambridge University Press, 2006.

49. Akira Onuki. Phase transition dynamics. Cambridge University Press, 2002.

50. Sicong Shan, Sung H Kang, Jordan R Raney, Pai Wang, Lichen Fang, Francisco Candido, Jennifer A Lewis, and Katia Bertoldi. Multistable architected materials for trapping elastic strain energy. Advanced Materials, 27(29):4296-4301, 2015.

51. Patrick Dorin, Jinki Kim, and Kon-Well Wang. Vibration energy harvesting system with coupled bistable modules. In Active and Passive Smart Structures and Integrated Systems XII, volume 10967, page 109670G. International Society for Optics and Photonics, 2019.

52. Narayanan Kidambi, Ryan L Harne, and KW Wang. Energy capture and storage in asymmetrically multistable modular structures inspired by skeletal muscle. Smart Materials and Structures, 26(8):085011, 2017.

53. Jordan R Raney, Neel Nadkarni, Chiara Daraio, Dennis M Kochmann, Jennifer A Lewis, and Katia Bertoldi. Stable propagation of mechanical signals in soft media using stored elastic energy. Proceedings of the National Academy of Sciences, 113(35):9722-9727, 2016.

54. Bolei Deng, Vincent Tournat, Pai Wang, and Katia Bertoldi. Anomalous collisions of elastic vector solitons in mechanical metamaterials. Physical review letters, 122(4):044101, 2019.

55. Bolei Deng, Chengyang Mo, Vincent Tournat, Katia Bertoldi, and Jordan R Raney. Focusing and mode separation of elastic vector solitons in a $2 \mathrm{~d}$ soft mechanical metamaterial. Physical review letters, 123(2):024101, 2019.

6. Hagen Kleinert and Verena Schulte-Frohlinde. Critical Properties of [Greek Letter Phi] 4theories. World Scientific, 2001.

57. Bolei Deng, Siqin Yu, Antonio E Forte, Vincent Tournat, and Katia Bertoldi. Characterization, stability, and application of domain walls in flexible mechanical metamaterials. Proceedings of the National Academy of Sciences, 2020.

58. Yoshimi Tanaka, Kentaro Ito, Toshiyuki Nakagaki, and Ryo Kobayashi. Mechanics of peristaltic locomotion and role of anchoring. Journal of the Royal Society Interface, 9(67):222233, 2012.

59. Langquan Shui, Liangliang Zhu, Zhe Yang, Yilun Liu, and Xi Chen. Energy efficiency of mobile soft robots. Soft Matter, 13(44):8223-8233, 2017.

60. FW Lewis, Suresh Jagannathan, and Aydin Yesildirak. Neural network control of robot manipulators and non-linear systems. CRC press, 1999. 


\title{
Supporting Information for Topological solitons make metamaterials crawl
}

\author{
Bolei Deng ${ }^{a, *}$, Mohamed Zanaty ${ }^{a, *}$, Antonio E. Forte ${ }^{a, b, c}$, Katia Bertoldi $^{a, \dagger}$ \\ ${ }^{a}$ Harvard John A. Paulson School of Engineering and Applied Sciences \\ Harvard University, Cambridge, Massachusetts 02138, USA \\ ${ }^{b}$ Department of Electronics, Information and Bioengineering, Politecnico di Milano, Milan, 20133, Italy \\ ${ }^{c}$ Department of Engineering, Kings College London, London, WC2R 2LS, UK \\ *These two authors contributed equally \\ ${ }^{\dagger}$ To whom correspondence should be addressed to bertoldi@seas.harvard.edu
}

November 21, 2021

\section{S1 Fabrication}

In this section, we provide details on the process used to fabricate the $1 \mathrm{D}$ chains and 2D networks of bistable units and to mount the actuators on the bistable units.

\section{S1.1 Bistable units}

The bistable unit of our system consists of four rigid crosses connected by intra-hinges (plastic shims) and stretched rubber bands. The unit is mounted either on pins (to study the propagation 
of transition waves) or rubber wheels (to demonstrate locomotion).

Each cross consists of four brackets $2 \times 2-2 \times 2$ (LEGO part 3956 - see Figure S1). The hinges are laser cut as rectangular shims with eight circular perforations out of a polyester plastic sheet (Artus Corporation, NJ - 0.01, brown) with thickness $\delta_{h}^{\text {intra }}=0.25 \mathrm{~mm}$, Youngs modulus $E=4.33 \mathrm{GPa}$ and Poisson's ratio $\nu=0.4$. The width of the rectangular hinge is $w_{h}^{\text {intra }}=22 \mathrm{~mm}$. We select the length of the shim such that the length of the intra-hinge is $l_{h}^{\text {intra }}=3.5 \mathrm{~mm}$.

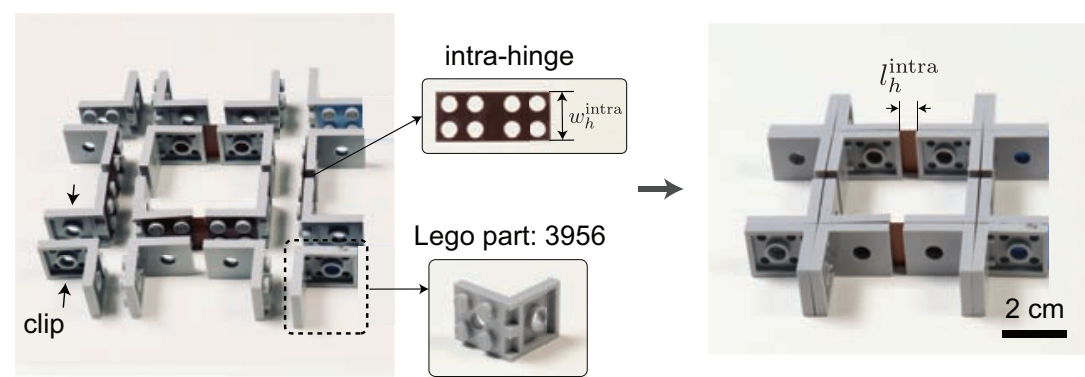

Figure S1: Assembly of a bistable unit.

After assembling the LEGO brackets and linking them with hinges, we add a rubber band on the top of the unit that connects all four crosses. For each cross, we use a support structure to hold the rubber band. The support structure consists of five rectangular rods and two square plates, which are laser cut out of an acrylic sheet of $4 \mathrm{~mm}$ thickness. The upper plate has four rectangular perforations on the corners and a fifth perforation on the top (4 $\mathrm{mm} \times 4 \mathrm{~mm})$ and the bottom plate has four rectangular perforations $(4 \mathrm{~mm} \times 4 \mathrm{~mm})$ on the corners and a circular hole with diameter of $0.2 \mathrm{~mm}$ in the center. The two plates are mounted on the bottom and top sides of the cross and connected using four rods $(25 \mathrm{~mm} \times 4 \mathrm{~mm} \times 4 \mathrm{~mm})$ on the corners. A post $(8 \mathrm{~mm} \times 4 \mathrm{~mm} \times 4 \mathrm{~mm})$ is added on the top of the upper plate. This post is used to keep the rubber band (Mobilon band $-110 \mathrm{~mm} \times 2 \mathrm{~mm} \times 0.3 \mathrm{~mm}$ ) in place. In Figure $\mathrm{S} 2$ we show the steps for the assembly of the support structure and the mounting of the rubber band. Note that the rubber band is transparent and it is highlighted by a red dashed line. 


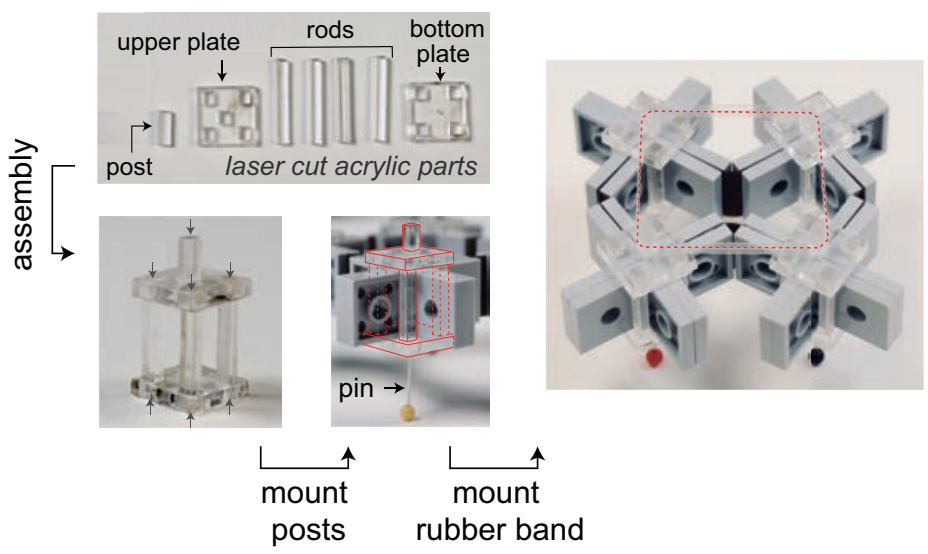

Figure S2: Assembly of the support structure and the rubber bands.

Finally, to study the propagation of transition waves, we attach a pin to the bottom plate of the support structure. Note that the bottom plate has a small hole of $0.2 \mathrm{~mm}$ diameter in which the pin fits. For the locomotion test, we replace the pins with a set of two wheels (see Figure S3). Each wheel has a rubber tire, which is mounted on a circular frame (LEGO parts: 30027a / 30028). Each pair of wheels are connected to a $2 \times 2$ Lego wheels holder (LEGO Part: 4600) and two $2 \times 2$ plate (LEGO Part: 3022). This assembly is glued to the bottom plate of the support structure using cyanoacrylate glue (Elmer's Products).

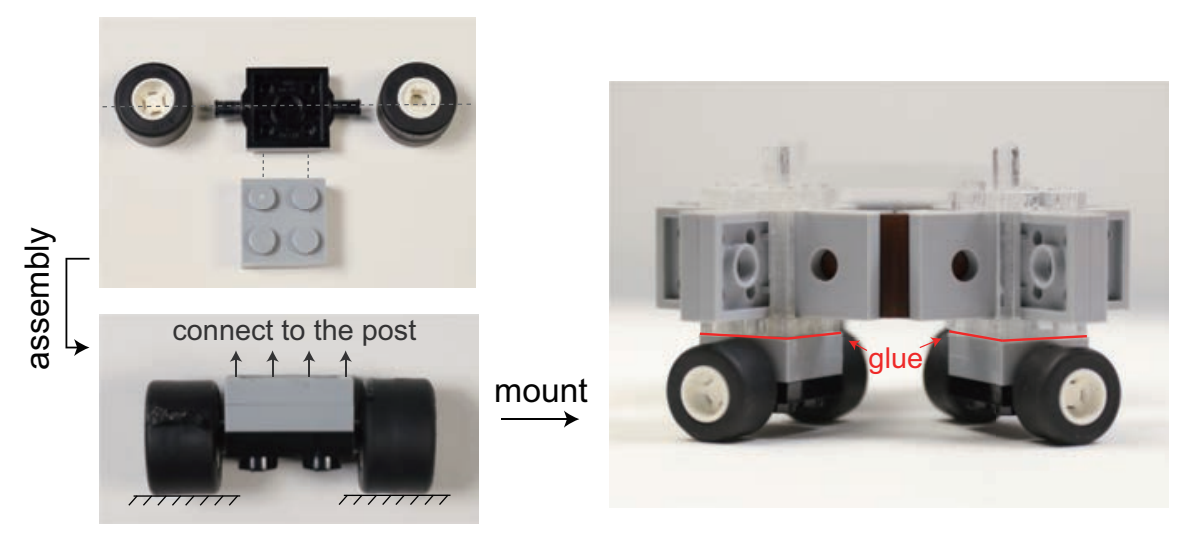

Figure S3: Mounting the rubber wheels. 


\section{S1.2 Network of bistable units}

Our system is a network of mechanically coupled bistable units. We use inter-hinges to couple the units (see Figure S4). These hinges are realized by laser cutting rectangular shapes out of polyester plastic sheets (Artus Corporation, NJ - 0.005, Blue) with thickness $\delta_{h}^{\text {inter }}=0.125 \mathrm{~mm}$, Young's modulus $E=4.33 \mathrm{GPa}$ and Poissons ratio $\nu_{h}=0.4$. The size of the rectangles is selected such that the spacing between unit cells is $l_{h}^{\text {inter }} \in[3,15] \mathrm{mm}$ (see Figure S4).

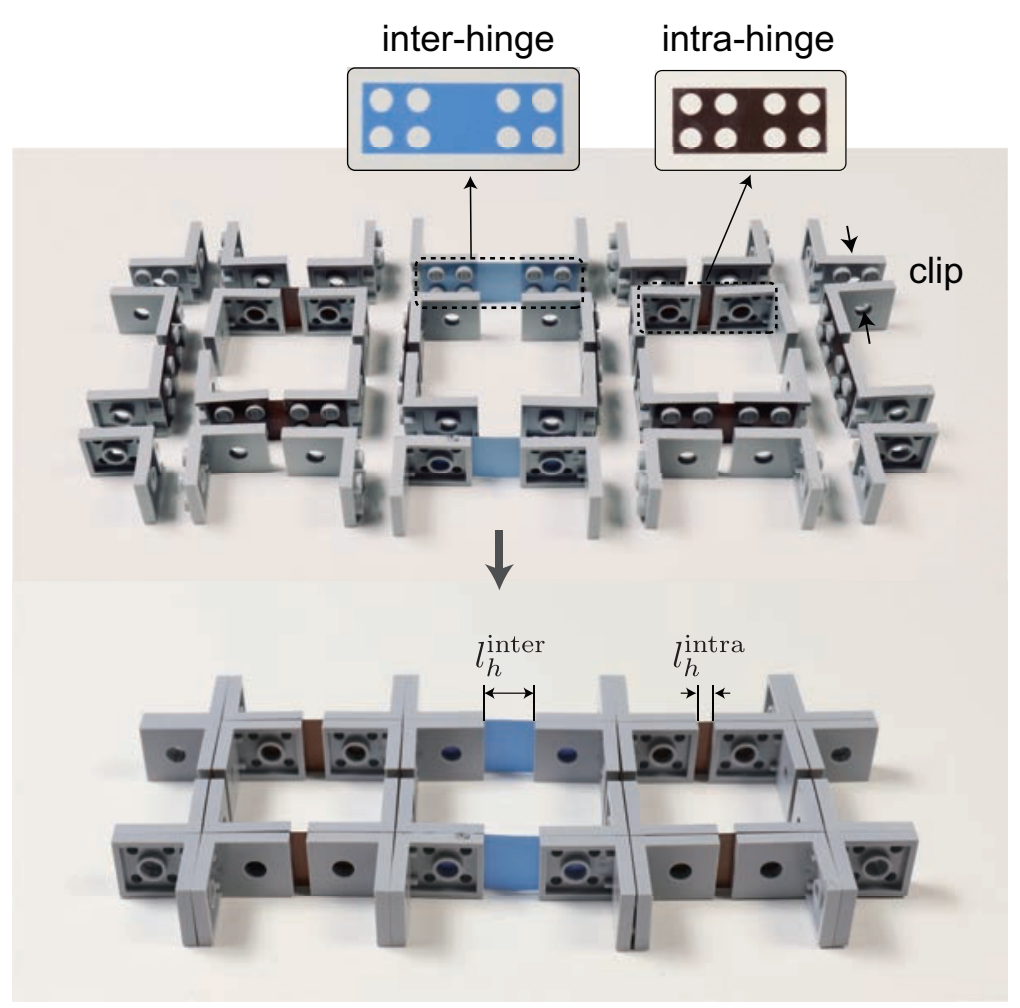

Figure S4: Assembly of a 1D network. The concept is demonstrate by the assembling of two units.

\section{S1.3 Actuators}

We use a linear micro-actuator (PQ12-R - Actuonix) to actuate our structures. The actuator is capable of producing a maximum force of $18 \mathrm{~N}$ and a stroke of $20 \mathrm{~mm}$. Since we need to impose a torque to rotate the crosses, we design a lever which can be mounted on the linear actuator on one side. On the other side, the lever is connected to the top post of the support structure of the 
crosses using rigid joints to convert the actuator output force into a torque (see Figure S5). We used two levers per actuator; each lever is laser cut out of transparent acrylic plates of thickness $4 \mathrm{~mm}$ and mounted on the actuator using screws and nuts.

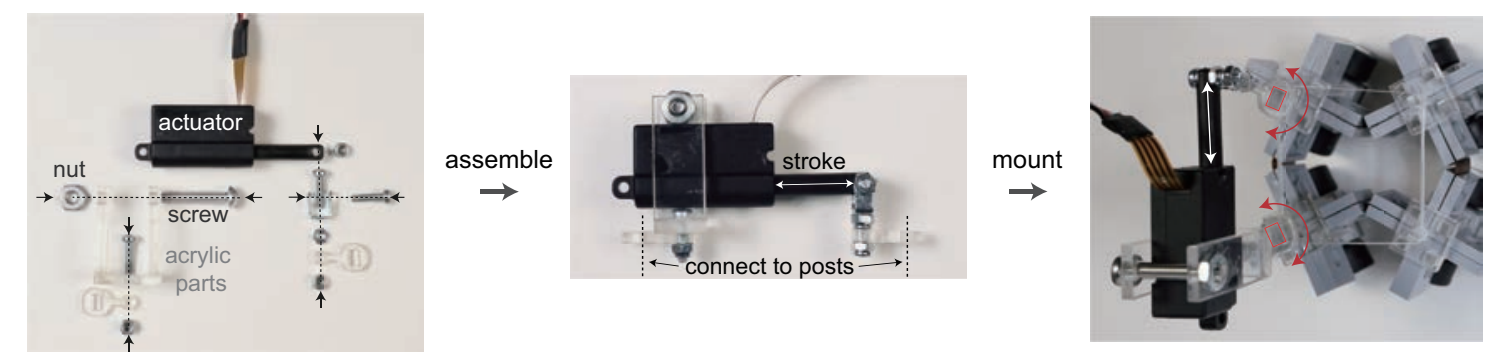

Figure S5: Assembly and mounting of the actuator.

A Uno Arduino kit is programmed to control the actuator. The actuator has three terminals, a black wire for the electrical ground, red wire connected to the $5 \mathrm{~V}$ power supply on the Arduino kit and a white wire for the control pulses. The stroke is programmed such that the actuator goes from zero to full stroke, then, holds its position for several seconds. After that, it retracts again from the full stroke to the zero position. In case of using multiple actuators for the same structure, we follow the same procedure for the assembly of each actuator and they are driven by the same Arduino kit. 


\section{S2 Testing}

In this section, we present the image processing used to track the rotation of the unit cells as well as the experiments conducted to characterize the bistable unit, the propagation of transition waves and locomotion. Note that here and thereafter, when considering $2 \mathrm{D}$ networks, we use the superscript $[i, j]$ to refer to quantities related to the $[i, j]$-th unit. Specifically, we use $u^{[i, j]}$, $v^{[i, j]}, \theta^{[i, j]}$ and $\beta^{[i, j]}$ to denote the displacements in $x$ and $y$ direction, the rotation of the crosses and the global rotation of the $[i, j]$-th unit, respectively. Differently, for $1 \mathrm{D}$ chains we use the superscript $j$ to refer to quantities related to the $j$-th unit.

\section{S2.1 Image processing}

To monitor the displacement and rotation of the bistable units, we use a high speed camera (SONY RX100) recording at $60 \mathrm{fps}$ and track four markers placed on the external arms of each unit (see Figure S6) via a superpixel-based method implemented in MATLAB. Specifically, after tracking the position of the four green markers on $[i, j]$-th unit at time $t\left(\left(x_{p}^{[i, j]}(t), y_{p}^{[i, j]}(t)\right)\right.$ with $p \in\{1,2,3,4\}$ ), the displacement and rotation of the considered two crosses are calculated as

$$
\begin{aligned}
& u_{k}^{[i, j]}(t)=\frac{1}{2}\left(x_{2 k-1}^{[i, j]}(t)-x_{2 k-1}^{[i, j]}(0)+x_{2 k}^{[i, j]}(t)-x_{2 k}^{[i, j]}(0)\right) \\
& v_{k}^{[i, j]}(t)=\frac{1}{2}\left(y_{2 k-1}^{[i, j]}(t)-y_{2 k-1}^{[i, j]}(0)+y_{2 k}^{[i, j]}(t)-y_{2 k}^{[i, j]}(0)\right) \\
& \theta_{k}^{[i, j]}(t)=\arcsin \left[\frac{\left(x_{2 k-1}^{[i, j]}(t)-x_{2 k-1}^{[i, j]}(0)\right)-\left(x_{2 k}^{[i, j]}(t)-x_{2 k}^{[i, j]}(0)\right)}{\sqrt{\left(x_{2 k}^{[i, j]}(0)-x_{2 k-1}^{[i, j]}(0)\right)^{2}+\left(y_{2 k}^{[i, j]}(0)-y_{2 k-1}^{[i, j]}(0)\right)^{2}}}\right]
\end{aligned}
$$

with $k=1,2$. Since the intra-hinges are much stiffer than the inter ones, we use the averaged displacements $u^{[i, j]}$ and $v^{[i, j]}$, the rigid body rotation of the entire unit $\beta^{[i, j]}$, together with the internal angle of the unit $\theta^{[i, j]}$ to describe the state of the $[i, j]$-unit,

$$
\begin{aligned}
& u^{[i, j]}=\frac{1}{2}\left(u_{1}^{[i, j]}+u_{2}^{[i, j]}\right), v^{[i, j]}=\frac{1}{2}\left(v_{1}^{[i, j]}+v_{2}^{[i, j]}\right), \\
& \beta^{[i, j]}=\frac{1}{2}\left(\theta_{1}^{[i, j]}+\theta_{2}^{[i, j]}\right) \text { and } \theta^{[i, j]}=\frac{1}{2}\left(\theta_{1}^{[i, j]}-\theta_{2}^{[i, j]}\right),
\end{aligned}
$$


where the minus sign in the expression of $\theta^{[i, j]}$ is introduced as neighbouring crosses always rotate in opposite directions.

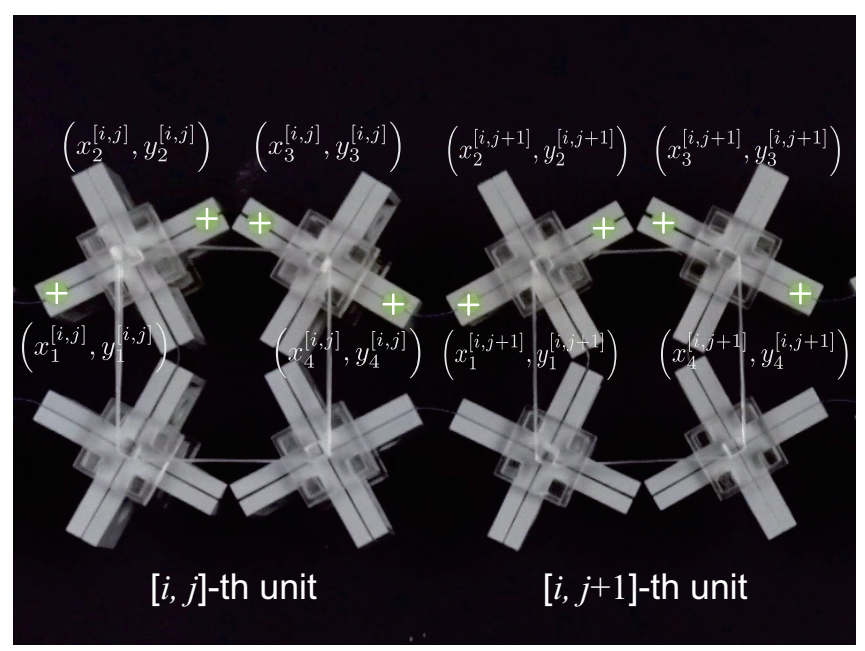

Figure S6: Digital image correlation analysis. Each bistable unit has four markers (green crosses) to be tracked.

\section{S2.2 Characterization of a bistable unit}

To calculate the strain energy of a bistable unit as a function of the rotation of its crosses $\theta_{j}$, we use an Instron machine (5960 series) equipped with a $50 \mathrm{kN}$ load cell. We mount the bistable unit to the testing machine as shown in Figure S7A and use levers and rigid joints to convert the linear displacement applied by the Instron into angular displacement (as discussed previously in Section S1.3).

In Figure S7B, we report the measured force, $F$, versus the applied displacement, $u_{\text {applied }}$. From these data we then calculate the associated potential energy as

$$
\tilde{\mathcal{E}}_{\text {intra }}\left(u_{\text {applied }}\right)=-\int F d u_{\text {applied }}
$$

where the integral is calculated numerically. As shown in Figure S7C, we find that the $\tilde{\mathcal{E}}_{\text {intra }}$ is characterized by two local minima at $u_{\text {applied }}=-13.5$ and $22.2 \mathrm{~mm}$, corresponding to two stable states. However, despite the symmetry of the two stable states, the energy minima at 
$u_{\text {applied }}=-13.5 \mathrm{~mm}$ is higher than the other one because of the gravity. To compensate for the effect of gravity, we calculate the gravitational energy as

$$
\mathcal{E}_{\text {grav }}\left(u_{\text {applied }}\right)=\sum_{i=1}^{4} \frac{m}{4} g v_{k}\left(u_{\text {applied }}\right)
$$

where $m=45 \mathrm{~g}$ is the mass of a unit cell (comprising four crosses), $g=9.81 \mathrm{~m} / \mathrm{s}^{2}$ is the gravitational acceleration and $v_{k}$ denotes the displacement in vertical direction of the $k$-th cross, which is a function of $u_{\text {applied }}$ and is obtained by tracking the green dots on the cross arms (see Section S2.1 for details). As shown in Figure S7D. the corrected energy

$$
\mathcal{E}^{\text {intra }}=\tilde{\mathcal{E}}_{\text {intra }}-\mathcal{E}_{\text {grav }}
$$

possesses two stable states with equal energy.

Finally, to obtain the strain energy as a function of the rotation of the crosses, we use image processing to extract $\theta^{[i, j]}$ (see Section S2.1 for details - see Figure S7E). By combining the data reported in Figures. S7E and S7D we obtain the strain energy of the bistable unit as function of the rotation of the crosses (see Figure S7F). The energy is characterized by two local minima at $\theta^{[i, j]}=-30.2^{\circ}$ and $29.8^{\circ}$. 


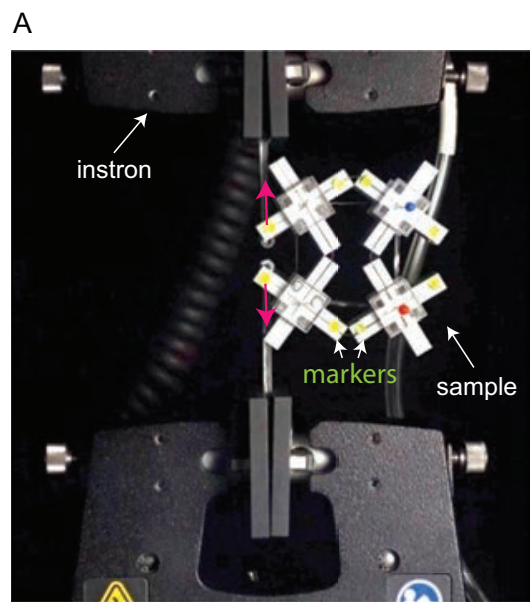

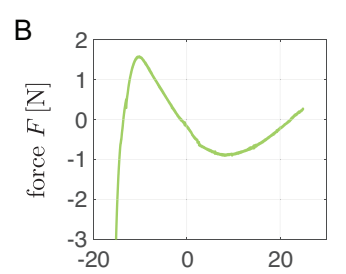

$$
\text { applied displ. } u_{\text {applied }}[\mathrm{mm}]
$$

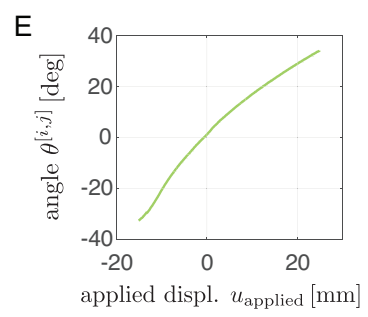

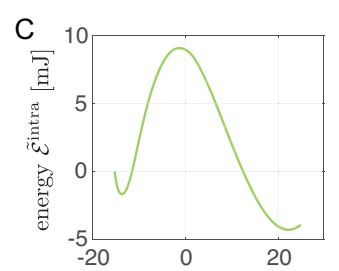

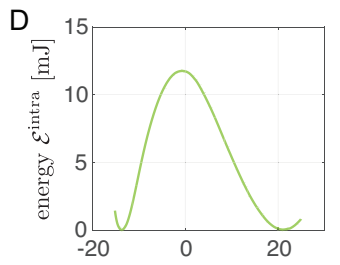

applied displ. $u_{\text {applied }}[\mathrm{mm}]$

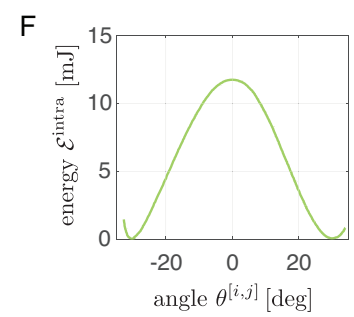

Figure S7: Measurement of the potential energy of the bistable unit. (A) measurement setup using the instron machine, (B) Measured force $F$ versus the displacement of the instron $u_{\text {applied }}$, (C) Strain energy $\mathcal{E}^{\text {intra }}$ calculated by the integration of the measured force $F$ with respect to displacement $u_{\text {applied }}$, (D) Strain energy $\mathcal{E}^{\text {intra }}$ on eliminating the effect of gravitational force, (E) Relation between the instron applied displacement $u_{\text {applied }}$ and the angular position of the unit $\theta^{[i, j]}$, (F) Calculated strain energy $\mathcal{E}^{\text {intra }}$ versus the angular position of the unit $\theta^{[i, j]}$.

\section{S2.3 Propagation of transition waves in a chain of bistable units mounted on pins}

To study the propagation of transition waves, we focus on a 1D chain of bistable units mounted on pins (to minimize friction). We place the structure on a horizontal rigid surface covered with black paper (kraft paper, Ruspepa). An actuator is mounted on the first unit (see Figure S5) and is used initiate a transition wave by pushing the first unit to the other stable configuration. The propagation of the waves is monitored using a high speed camera (SONY RX 100V) placed above the samples that records at $60 \mathrm{fps}$. From the recorded movies we then extract the position and rotation of each bistable unit using the image processing techniques discussed in Section S2.1. 


\section{S2.4 Locomotion of a network of bistable units mounted on wheels}

Our bistable networks move when mounted on wheels. To characterize such motion we place the wheeled network on a horizontal rigid surface covered with black paper. Actuators mounted on selected units are used to initiate transition waves by pushing the units to the other stable configuration. As shown in Figure S8, all our tests are monitored using a high speed camera (SONY RX 100V) positioned above the sample. The position of each bistable unit is then extracted from the recorded movies using the image processing technique discussed in Section S2.1.

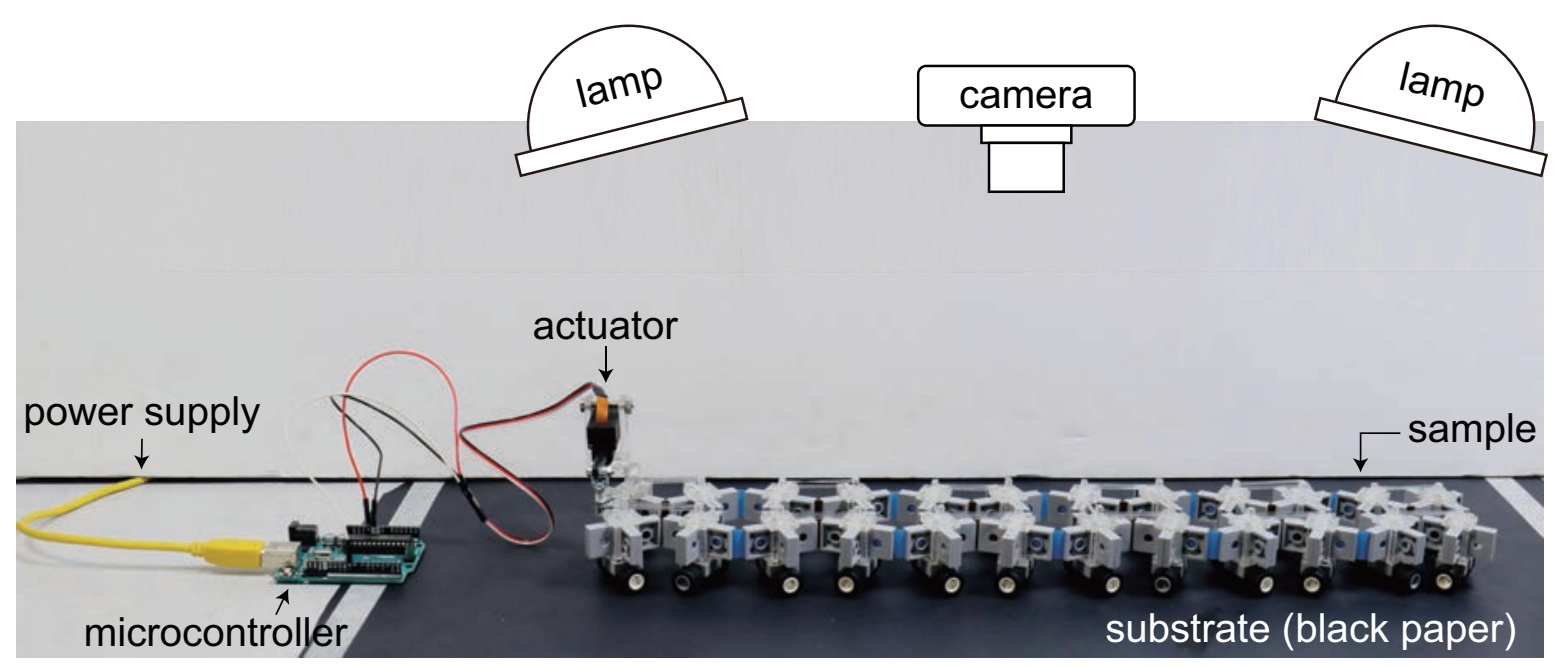

Figure S8: Experimental characterization setup. A micro-controller is used to power the actuator and a camera is set up to track the movement of $1 \mathrm{D}$ bistable chain crawling robot. 


\section{S2.5 Additional experimental results}
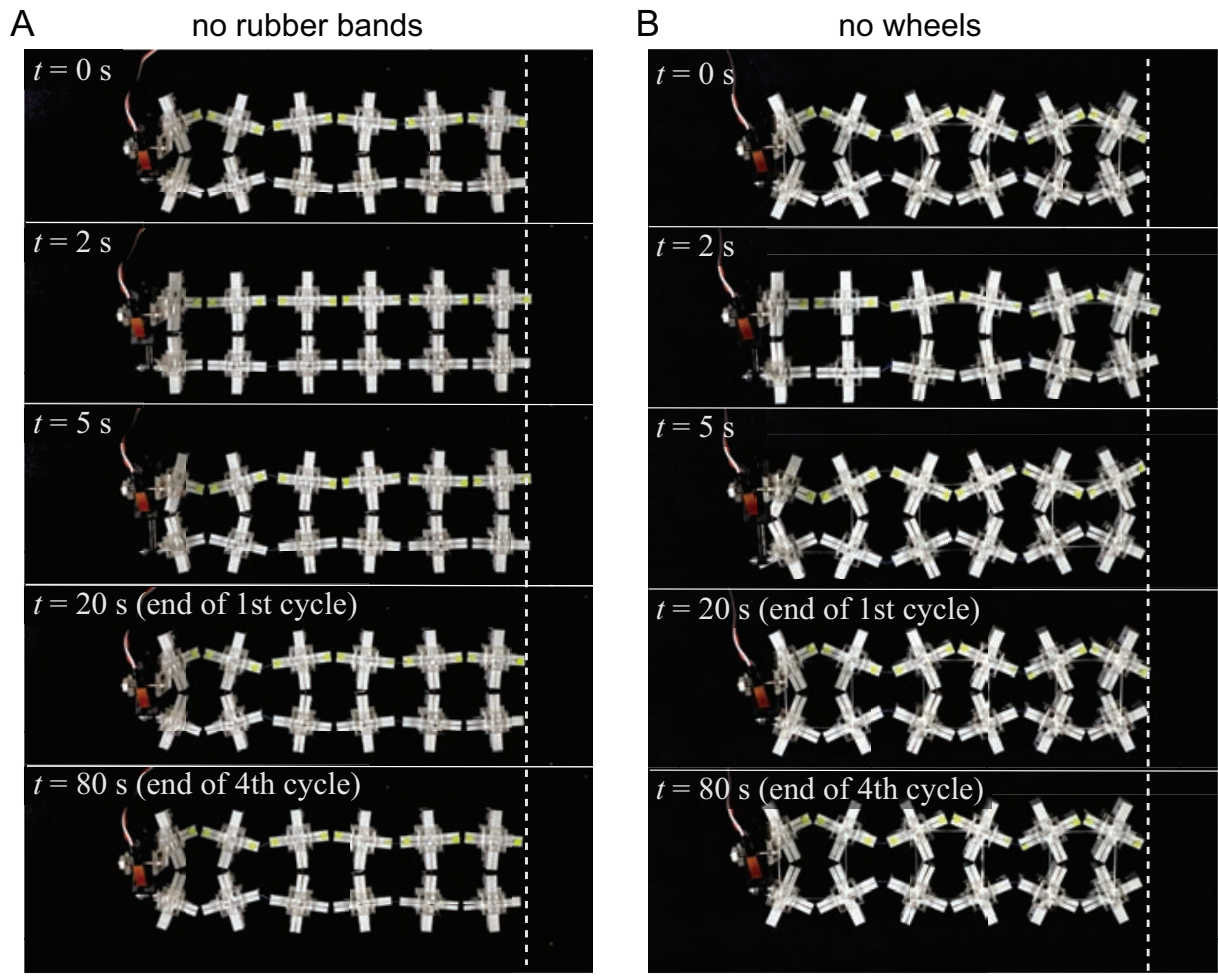

Figure S9: Additional experimental results. Locomotion of a chain with $N_{x}=3$ and $l_{h}^{\text {inter }}=6 \mathrm{~mm}$ in the absence of (A) rubber bands and (B) wheels. (A) When the rubber bands are removed, the unit cell becomes monostable. While the monostable system has been shown to supports solitary pulses, these cannot be initiated by slow input signal provided by the linear actuator. Therefore, $d_{\text {step }}=0 \mathrm{~mm}$, i.e. locomotion is hindered. (B) When the wheels are removed and pins are used to support the structure, the rotational component of the topological solitons can not be effectively harnessed to manipulate friction. Once again, locomotion is hindered. 


\section{S3 Mathematical Model}

\section{S3.1 Bistable unit cell}

Our bistable unit consists of four LEGO-crosses connected by flexible intra-hinges and prestretched rubber bands (see Figure S10). To capture the response of the $[i, j]$ unit cell, we model $(i)$ the crosses as cross-shaped rigid bodies with arm length $l_{a} \mathrm{~mm}$ that rotate by the same amount $\theta^{[i, j]}$ (with neighboring units rotating in opposite direction); (ii) the hinges as linear rotational springs with stiffness $k_{\theta}$; and (iii) the rubber bands as four linear springs with stiffness $k_{b}$ and rest length $l_{b}=0.764 l_{p}\left(l_{p}\right.$ being the distance the posts to which the rubber bands are attached when the crosses are aligned - i.e. $\theta^{[i, j]}=0$ ).

A

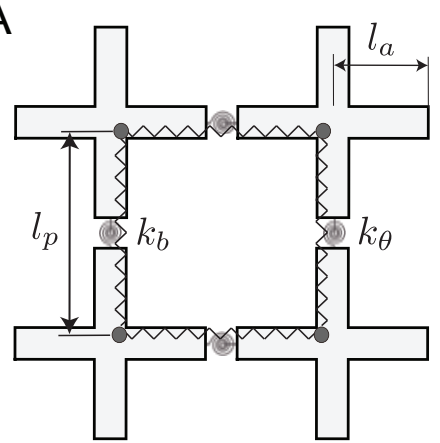

$\mathrm{B}$

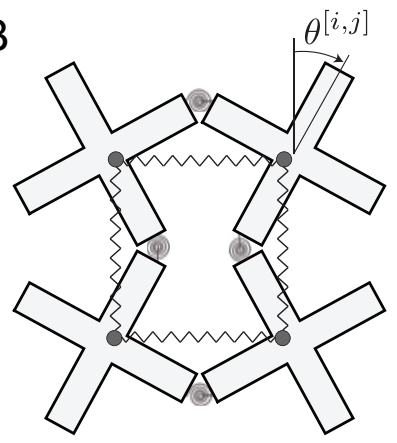

C

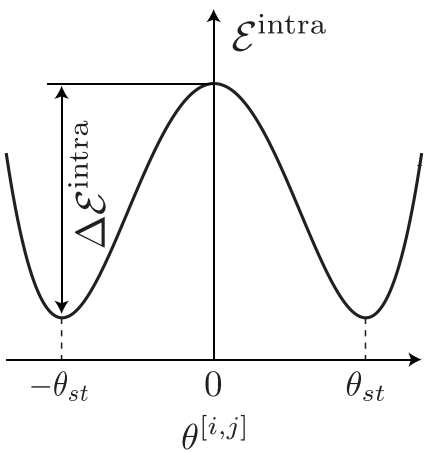

Figure S10: Mathematical model of a unit cell. (A,B) Schematics of a bistable unit at different angular positions $\theta_{[i, j]}$, (C) Energy profile of the bistable unit with energy barrier $\Delta \mathcal{E}$.

Under the assumptions listed above, the total energy of the unit cell can be expressed as

$$
\mathcal{E}^{\text {intra }}\left(\theta^{[i, j]}\right)=2 k_{b}\left(l_{p} \cos \theta^{[i, j]}-l_{b}\right)^{2}+8 k_{\theta}\left(\theta^{[i, j]}\right)^{2},
$$

which is a non-convex function with two symmetric wells at $\pm \theta_{s t}$ (see Figure S10C). By approximating $\sin \theta^{[i, j]} \approx \theta^{[i, j]}$ and $\cos \theta^{[i, j]} \approx 1-\left(\theta^{[i, j]}\right)^{2} / 2$, the location of the two minima are obtained as

$$
\theta_{s t}= \pm \sqrt{2-\frac{2 l_{b}}{l_{p}}-\frac{8 k_{\theta}}{l_{p}^{2} k_{b}}} .
$$

Further, since $\theta^{[i, j]}=0$ is a local maximum (see Figure S10C), the energy barrier to overcome 
to move from one stable configuration to the other one is

$$
\begin{aligned}
\Delta \mathcal{E}^{\text {intra }} & =\mathcal{E}^{\text {intra }}(0)-\mathcal{E}^{\text {intra }}\left(\theta_{s t}\right) \\
& =2 l_{p}^{2} k_{b}\left[\left(1-\frac{l_{b}}{l_{p}}\right)^{2}-\left(\cos \theta_{s t}-\frac{l_{b}}{l_{p}}\right)^{2}\right]-8 k_{\theta} \theta_{s t}^{2} \\
& \approx \frac{1}{2} k_{b} l_{p}^{2} \theta_{s t}^{4} .
\end{aligned}
$$

\section{S3.2 Network of bistable units}

In our experiments we consider networks comprising $N_{x} \times N_{y}$ bistable units connected by interhinges. Such connections are captured in our analyses by two linear springs: a longitudinal spring with stiffness $k_{l}$ and a shearing spring with stiffness $k_{s}$ (see Figure S11 - note that the bending rigidity of the inter-hinges is very small and, therefore, is neglected). Four degrees of freedom are assigned to the $[i, j]$-th unit cell (see Figure S11): $(i)$ the displacements in $x$ direction, $u^{[i, j]}$; (ii) the displacements in $y$ direction $y, v^{[i, j]}$; (iii) the global rotation of the unit cell, $\beta^{[i, j]} ;(i v)$ the internal rotation of the four crosses within the unit, $\theta^{[i, j]}$. Under these assumptions, the kinetic and elastic energy of a network comprising $N_{x} \times N_{y}$ units can be written as

$$
\mathcal{E}_{\text {kin }}=\sum_{i=1}^{N_{y}} \sum_{j=1}^{N_{x}} \mathcal{E}_{\text {kin }}^{[i, j]}
$$

and

$$
\mathcal{E}_{\text {ela }}=\left(\sum_{i=1}^{N_{y}} \sum_{j=1}^{N_{x}} \mathcal{E}^{\text {intra }}\left(\theta^{[i, j]}\right)+\sum_{p=1}^{N_{\text {inter }}} \mathcal{E}_{p}^{\text {inter }}\right)
$$

where $\mathcal{E}_{\text {kin }}^{[i, j]}$ denotes the kinetic energy of the $[i, j]$-th unit, $\mathcal{E}^{\text {intra }}\left(\theta^{[i, j]}\right)$ is the elastic energy of the $[i, j]$-th unit that is given by Eq. (S6), $\mathcal{E}_{p}^{\text {inter }}$ represents the elastic energy of the $p$-th connection between neighboring units and $N_{\text {inter }}$ is the total number of inter-unit connections (note that $N_{\text {inter }}$ is equal to half the number of inter-hinges in our system, since each connection is realized using a pair of inter-hinges). Specifically, the kinetic energy of the $[i, j]$-th unit cell can be written as

$$
\mathcal{E}_{\text {kin }}^{[i, j]}=\frac{1}{2} m\left(\dot{u}^{[i, j]}\right)^{2}+\frac{1}{2} m\left(\dot{v}^{[i, j]}\right)^{2}+\frac{1}{2} J_{\beta}\left(\dot{\beta}^{[i, j]}\right)^{2}+\frac{1}{2} J_{\theta}\left(\dot{\theta}^{[i, j]}\right)^{2},
$$


where $\dot{f}$ indicates the time derivative $d f / d t$ for arbitrary variable $f, m$ is the mass of each unit and $J_{\theta}$ and $J_{\beta}$ are the rotational inertia associated with $\beta^{[i, j]}$ and $\theta^{[i, j]}$, respectively.

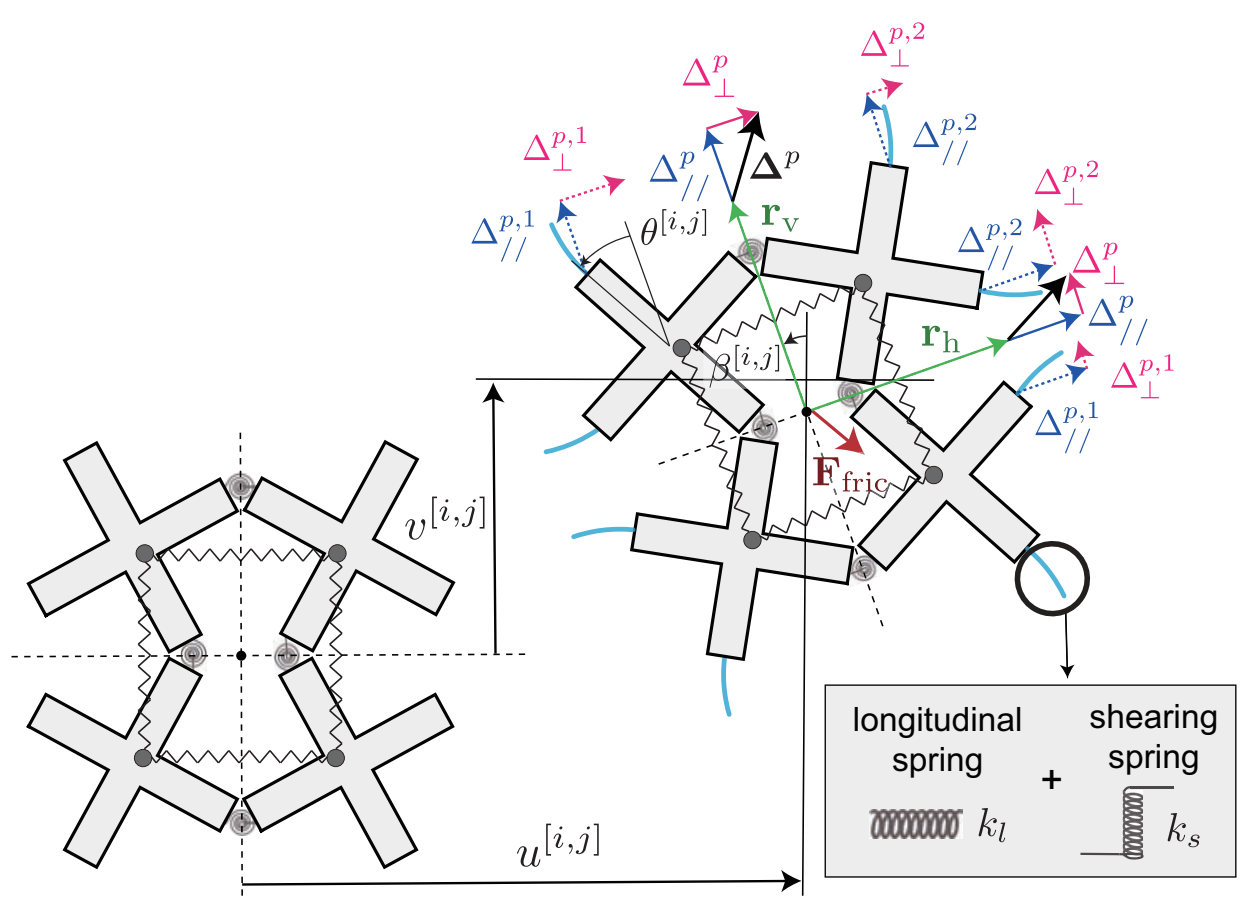

Figure S11: General mathematical model of the network. A schematic of a unit cell in the network and the interactions with its neighbours.

As for the elastic energy associated to the deformation of the inter-hinges connecting neighboring units, we have

$$
\mathcal{E}_{p}^{\text {inter }}=\frac{1}{2} k_{l}\left[\left(\Delta_{/ /}^{p, 1}\right)^{2}+\left(\Delta_{/ /}^{p, 2}\right)^{2}\right]+\frac{1}{2} k_{s}\left[\left(\Delta_{\perp}^{p, 1}\right)^{2}+\left(\Delta_{\perp}^{p, 2}\right)^{2}\right]
$$

where $\Delta_{/ /}^{p, j}$ and $\Delta_{\perp}^{p, j}$ are the extensions of the $j$-th (with $j=1,2$ ) longitudinal and shearing springs in the $p$-th interconnection between neighboring units (see Figure S11). These extensions can be calculated as 
- Connections between units $[i, j]$ and $[i, j+1]$ : for this case we have

$$
\begin{aligned}
& \Delta_{/ /}^{p, 1}=\Delta_{/ /}^{p}+l_{a}\left(1-\cos \theta^{[i, j]}-\sin \theta^{[i, j]}\right)\left(\beta^{[i, j+1]}-\beta^{[i, j]}\right) \\
& \Delta_{/ /}^{p, 2}=\Delta_{/ /}^{p}-l_{a}\left(1-\cos \theta^{[i, j]}-\sin \theta^{[i, j]}\right)\left(\beta^{[i, j+1]}-\beta^{[i, j]}\right) \\
& \Delta_{\perp}^{p, 1}=\Delta_{\perp}^{p}+l_{a}\left(\sin \theta^{[i, j+1]}-\sin \theta^{[i, j]}\right) \\
& \Delta_{\perp}^{p, 2}=\Delta_{\perp}^{p}-l_{a}\left(\sin \theta^{[i, j+1]}-\sin \theta^{[i, j]}\right)
\end{aligned}
$$

with

$$
\left(\begin{array}{c}
\Delta_{/ /}^{p} \\
\Delta_{\perp}^{p}
\end{array}\right)=\left(\begin{array}{cc}
\cos \beta^{[i, j]} & \sin \beta^{[i, j]} \\
-\sin \beta^{[i, j]} & \cos \beta^{[i, j]}
\end{array}\right) \Delta^{p}
$$

and

$$
\begin{aligned}
\boldsymbol{\Delta}^{p}= & \left(\begin{array}{c}
u^{[i, j+1]}-u^{[i, j]} \\
v^{[i, j+1]}-v^{[i, j]}
\end{array}\right)+\left[\mathbf{r}_{\mathrm{h}}\left(\theta^{[i, j+1]}, \beta^{[i, j+1]}\right)-\mathbf{r}_{\mathrm{h}}\left(\theta_{s t}, 0\right)\right] \\
& +\left[\mathbf{r}_{\mathrm{h}}\left(\theta^{[i, j]}, \beta^{[i, j]}\right)-\mathbf{r}_{\mathrm{h}}\left(\theta_{s t}, 0\right)\right]
\end{aligned}
$$

where $\mathbf{r}_{\mathrm{h}}\left(\theta^{[i, j]}, \beta^{[i, j]}\right)$ denotes the vector that connects the center of the $[i, j]$-unit cell rigid unit to the middle point between the two inter-hinges (see Figure S11)

$$
\mathbf{r}_{\mathrm{h}}\left(\theta^{[i, j]}, \beta^{[i, j]}\right)=2 l_{a} \cos \theta^{[i, j]}\left(\begin{array}{l}
\cos \beta^{[i, j]} \\
\sin \beta^{[i, j]}
\end{array}\right) .
$$

- Connections between units $[i, j]$ and $[i+1, j]$ : for this case we have

$$
\begin{aligned}
& \Delta_{/ /}^{p, 1}=\Delta_{/ /}^{p}-l_{a}\left(1-\cos \theta^{[i, j]}+\sin \theta^{[i, j]}\right)\left(\beta^{[i+1, j]}-\beta^{[i, j]}\right) \\
& \Delta_{/ /}^{p, 2}=\Delta_{/ /}^{p}+l_{a}\left(1-\cos \theta^{[i, j]}+\sin \theta^{[i, j]}\right)\left(\beta^{[i+1, j]}-\beta^{[i, j]}\right) \\
& \Delta_{\perp}^{p, 1}=\Delta_{\perp}^{p}-l_{a}\left(\sin \theta^{[i+1, j]}-\sin \theta^{[i, j]}\right) \\
& \Delta_{\perp}^{p, 2}=\Delta_{\perp}^{p}+l_{a}\left(\sin \theta^{[i+1, j]}-\sin \theta^{[i, j]}\right)
\end{aligned}
$$

with

$$
\left(\begin{array}{c}
\Delta_{/ /}^{p} \\
\Delta_{\perp}^{p}
\end{array}\right)=\left(\begin{array}{cc}
-\sin \beta^{[i, j]} & \cos \beta^{[i, j]} \\
\cos \beta^{[i, j]} & \sin \beta^{[i, j]}
\end{array}\right) \Delta^{p}
$$


and

$$
\begin{aligned}
\boldsymbol{\Delta}^{p}= & \left(\begin{array}{c}
u^{[i+1, j]}-u^{[i, j]} \\
v^{[i+1, j]}-v^{[i, j]}
\end{array}\right)+\left[\mathbf{r}_{\mathbf{v}}\left(\theta^{[i+1, j]}, \beta^{[i+1, j]}\right)-\mathbf{r}_{\mathbf{v}}\left(\theta_{s t}, 0\right)\right] \\
& -\left[\mathbf{r}_{\mathbf{v}}\left(\theta^{[i, j]}, \beta^{[i, j]}\right)-\mathbf{r}_{\mathbf{v}}\left(\theta_{s t}, 0\right)\right]
\end{aligned}
$$

where $\mathbf{r}_{\mathbf{v}}\left(\theta^{[i, j]}, \beta^{[i, j]}\right)$ denotes the vector that connects the center of the $[i, j]$-unit cell to the middle point between the two inter-hinges (see Figure S11)

$$
\mathbf{r}_{\mathrm{v}}\left(\theta^{[i, j]}, \beta^{[i, j]}\right)=2 l_{a} \cos \theta^{[i, j+1]}\left(\begin{array}{c}
-\sin \beta^{[i, j]} \\
\cos \beta^{[i, j]}
\end{array}\right) .
$$

The equation of motion for the $[i, j]$-th unit cell are then obtained via the Euler-Lagrange equations as

$$
\begin{aligned}
m \ddot{u}^{[i, j]} & =\frac{\partial \mathcal{L}}{\partial u^{[i, j]}} \\
m \ddot{v}^{[i, j]} & =\frac{\partial \mathcal{L}}{\partial v^{[i, j]}} \\
J_{\beta} \ddot{\beta}^{[i, j]} & =\frac{\partial \mathcal{L}}{\partial \beta^{[i, j]}} \\
J_{\theta} \ddot{\theta}^{[i, j]} & =\frac{\partial \mathcal{L}}{\partial \theta^{[i, j]}}
\end{aligned}
$$

where $\mathcal{L}=\mathcal{E}_{\text {kin }}-\mathcal{E}_{\text {ela }}$. Finally, to account for the energy dissipated due to the sliding of the structure on the surface and well as to internal damping, we add friction terms so that the equations of motion become

$$
\begin{aligned}
m \ddot{u}^{[i, j]} & =\frac{\partial \mathcal{L}}{\partial u^{[i, j]}}+\mathbf{F}_{\text {fric }}^{[i, j]} \cdot \mathbf{e}_{x} \\
m \ddot{v}^{[i, j]} & =\frac{\partial \mathcal{L}}{\partial v^{[i, j]}}+\mathbf{F}_{\text {fric }}^{[i, j]} \cdot \mathbf{e}_{y} \\
J_{\beta} \ddot{\beta}^{[i, j]} & =\frac{\partial \mathcal{L}}{\partial \beta^{[i, j]}} \\
J_{\theta} \ddot{\theta}^{[i, j]} & =\frac{\partial \mathcal{L}}{\partial \theta^{[i, j]}}+M_{\text {fric }}^{[i, j]}
\end{aligned}
$$

where $\mathbf{F}_{\text {fric }}^{[i, j]}$ and $M_{\text {fric }}^{[i, j]}$ denotes the frictional force and moment between the unit and the sub- 
strate, which we determine as

- Unit cell with pins attached to its base. In this case the frictional force is isotropic and can be written as

$$
\mathbf{F}_{\text {fric }}^{[i, j]}=-m g \mu_{\text {pin }} \operatorname{sign}\left(\begin{array}{c}
\dot{u}^{[i, j]} \\
\dot{v}^{[i, j]}
\end{array}\right), \text { and } M_{\text {fric }}^{[i, j]}=0
$$

where $\mu_{\text {pin }}$ is the friction coefficient between the pin and the substrate.

- Unit cell with wheels attached to its base. In this case the frictional force for each unit depends on $\beta^{[i, j]}, \theta^{[i, j]}$ and the direction of motion and can be written as

$$
\begin{aligned}
\mathbf{F}_{\text {fric }}^{[i, j]} & =\left(\begin{array}{cc}
\cos \beta^{[i, j]} & \sin \beta^{[i, j]} \\
-\sin \beta^{[i, j]} & \cos \beta^{[i, j]}
\end{array}\right)\left(\begin{array}{l}
-m g \mu_{s}\left|\sin \theta^{[i, j]}\right| \operatorname{sign}\left(\dot{u}_{/ /}^{[i, j]}\right) \\
-m g \mu_{s}\left|\cos \theta^{[i, j]}\right| \operatorname{sign}\left(\dot{u}_{\perp}^{[i, j]}\right)
\end{array}\right), \\
M_{\text {fric }}^{[i, j]} & =m g \mu_{s} d_{\mathrm{wh}}
\end{aligned}
$$

where $\mu_{s}$ is the coefficient of friction measured when sliding the wheels on the substrate and $d_{\mathrm{wh}}=20 \mathrm{~mm}$ denotes the distance between two wheels under the same cross. Note that here we neglect rolling resistance, so that the frictional coefficient for a wheel moving at an angle $\alpha$ with respect to the rolling direction is given by $\mu_{s} \cos \alpha$. Further, $\dot{u}_{/ /}^{[i, j]}$ and $\dot{u}_{\perp}^{[i, j]}$ are the components of velocity of the unit along the principal axis of the unit cell (indicated by black dash lines in Figure S11), which can be obtained as

$$
\left(\begin{array}{l}
\dot{u}_{/ i, j]}^{[i} \\
\dot{u}_{\perp}^{[i, j]}
\end{array}\right)=\left(\begin{array}{cc}
\cos \beta^{[i, j]} & -\sin \beta^{[i, j]} \\
\sin \beta^{[i, j]} & \cos \beta^{[i, j]}
\end{array}\right)\left(\begin{array}{l}
\dot{u}^{[i, j]} \\
\dot{v}^{[i, j]}
\end{array}\right)
$$

For a system comprising $N_{x} \times N_{y}$ units Eqs. (S22) provide a system with $4 N_{x} N_{y}$ coupled differential equations in $u^{[i, j]}, v^{[i, j]}, \beta^{[i, j]}$ and $\theta^{[i, j]}$ that we numerically integrate using the 4 th order Runge-Kutta method (via the Matlab function ode45). 


\section{S3.3 Parameter Identification}

To connect the discrete model to our experimental sample, we need to estimate the mass of the unit cell $(m)$, the rotational inertia $\left(J_{\beta}\right.$ and $\left.J_{\theta}\right)$, the spring stiffnesses $\left(k_{l}, k_{s}, k_{b}\right.$ and $\left.k_{\theta}\right)$ and the friction coefficients $\left(\mu_{p i n}\right.$ and $\left.\mu_{s}\right)$ as well as the geometric parameters $l_{a}$ and $l_{p}$.

Geometric parameters $l_{a}$ and $l_{p}$. For the system considered in this study the arm length of the rigid crosses is $l_{a}=19 \mathrm{~mm}$ and the distance between the posts to which the rubber bands are attached when the crosses are aligned (i.e. or $\theta^{[i, j]}=0$ ) is $l_{p}=36 \mathrm{~mm}$.

Mass $m$. The mass of each bistable unit is measured as $m=45 \mathrm{~g}$.

Rotational inertias $J_{\beta}$ and $J_{\theta}$. To obtain $J_{\beta}$, we first note that the kinetic energy associated with $\beta^{[i, j]}$ can be expressed as (see Figure S12A)

$$
\mathcal{E}_{\text {kin }, \beta}^{[i, j]}=4\left[\frac{1}{2} m\left(u_{\text {cross }}^{2}+v_{\text {cross }}^{2}\right)\left(\dot{\beta}^{[i, j]}\right)^{2}+\frac{1}{2} J_{\text {cross }}\left(\dot{\beta}^{[i, j]}\right)^{2}\right]=\frac{1}{2} J_{\beta}\left(\dot{\beta}^{[i, j]}\right)^{2},
$$

where $u_{\text {cross }}=l_{a} \cos \theta^{[i, j]}, v_{\text {cross }}=l_{a} \cos \theta^{[i, j]}$ and $J_{\text {cross }}=6.05 \times 10^{-4} \mathrm{~g} \cdot \mathrm{m}^{2}$ is the rotational inertia of a cross with respect to its center of mass [1]. It follows that

$$
J_{\beta}=8 m l_{a}^{2}\left(\cos \theta^{[i, j]}\right)^{2}+4 J_{\text {cross }}
$$

In an attempt to simplify our calculations, we assume that $\theta^{[i, j]} \approx \theta_{s t}$, so that

$$
J_{\beta} \approx 8 m l_{a}^{2}\left(\cos \theta_{s t}\right)^{2}+4 J_{\text {cross }}=0.116 \mathrm{~g} \cdot \mathrm{m}^{2} .
$$

Next, to obtain $J_{\theta}$ we note that the kinetic energy associated to $\theta^{[i, j]}$ can be is expressed as (see Figure S12B)

$$
\mathcal{E}_{\text {kin }, \theta}^{[i, j]}=4\left[\frac{1}{2} m\left(\dot{u}_{\text {cross }}^{2}+\dot{v}_{\text {cross }}^{2}\right)+\frac{1}{2} J_{\text {cross }}\left(\dot{\theta}^{[i, j]}\right)^{2}\right]=\frac{1}{2} J_{\theta}\left(\dot{\theta}^{[i, j]}\right)^{2}
$$

It follows that

$$
J_{\theta}=8 m l_{a}^{2}\left(\sin \theta^{[i, j]}\right)^{2}+4 J_{\text {cross }}
$$

and again, to simplify the calculations, we neglect the dependency from $\theta^{[i, j]}$ and approximate 
it as

$$
J_{\theta} \approx 8 m l_{a}^{2} \sin ^{2} \theta_{s t}+4 J_{\text {cross }}=0.065 \mathrm{~g} \cdot \mathrm{m}^{2}
$$

Finally, we want to emphasize that, since in our system the elastic energy denotes the kinetic one, the approximations introduced to estimate $J_{\theta}$ and $J_{\beta}$ do not affect the results.
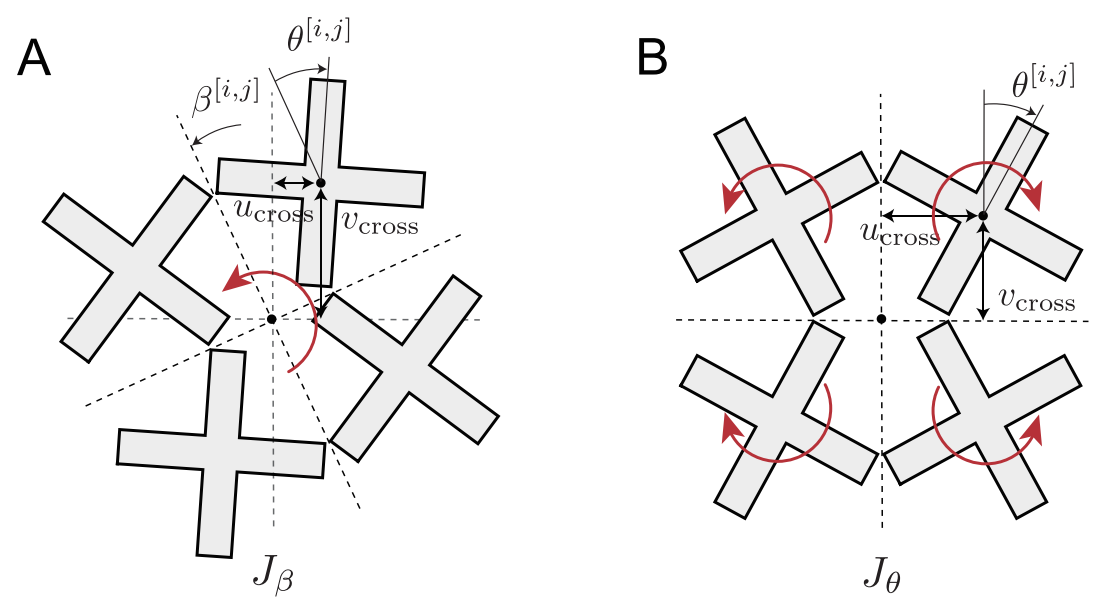

Figure S12: Rotational inertia of a unit cell. Inertia associated with $\beta^{[i, j]}$ and $\theta^{[i, j]}$ (A) $\beta^{[i, j]}>0$, (B) $\beta^{[i, j]}=0$.

Torsional stiffness $k_{\theta}$. To measure the rotational stiffness of the intra-hinges that connect the four crosses forming the bistable unit, we conduct a uniaxial test using an Instron machine. We follow the same procedure described in Section S2.2, but the unit is tested with no rubber band. The estimated strain energy due to the deformation of the four hinges as a function of internal angle $\theta$ is reported in Figure $\mathbf{S} 13$. 


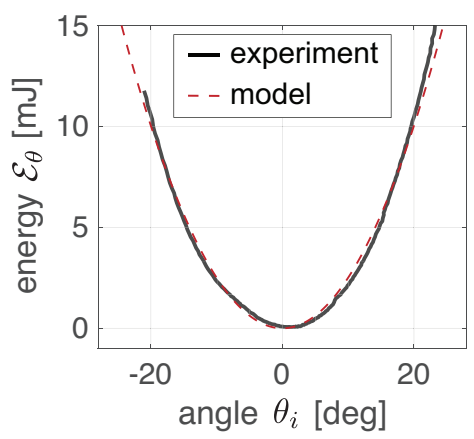

Figure S13: Characterization of $k_{\theta}$. The elastic energy $\mathcal{E}_{\theta}$ of the intra-hinges as a function of the angular position $\theta_{i}$.

By comparing the experimental curve with the predicted one

$$
\mathcal{E}_{\theta}=8 k_{\theta} \theta^{2}
$$

we find that $k_{\theta}=0.0103 \mathrm{~N} \cdot \mathrm{m}$.

Stiffness $k_{b}$ and initial length $l_{b}$. In our experiments, we used a rubber of a rectangular cross section of $2 \mathrm{~mm} \times 0.3 \mathrm{~mm}$ (cross section area $A_{b}=0.6 \mathrm{~mm}^{2}$ ) and an initial length of $110 \mathrm{~mm}$, so that $l_{b}=110 / 4=27.5 \mathrm{~mm}$. We measure the stiffness of the rubber bands using the Instron. The rubber band is clamped at two ends resulting in a rest length of $l_{b}^{\text {ins }}=50 \mathrm{~mm}$ and is then stretched by applying a displacement $x_{b}^{\text {ins }}$ while recording the force, $F_{b}^{\text {ins }}$, with a force sensor. The Young's modulus of the rubber band is then calculated as,

$$
E_{b}=\frac{F_{b}^{\text {ins }} l_{b}^{\text {ins }}}{A_{b} x_{b}^{\text {ins }}}=7.0 \mathrm{MPa}
$$

To ensure the consistency of our measured values, the experiment was repeated or 15 randomly selected rubber bands as highlighted in Figure S14. The stiffness of the linear spring $k_{b}$ that is used to model rubber band in discrete model can be obtained via

$$
k_{b}=\frac{E_{b} A_{b}}{l_{b}}=305 \mathrm{~N} / \mathrm{m}
$$

The experiment was repeated or 15 randomly selected rubber bands as highlighted in Figure S14 to verify the consistency of the measured stiffness values. 

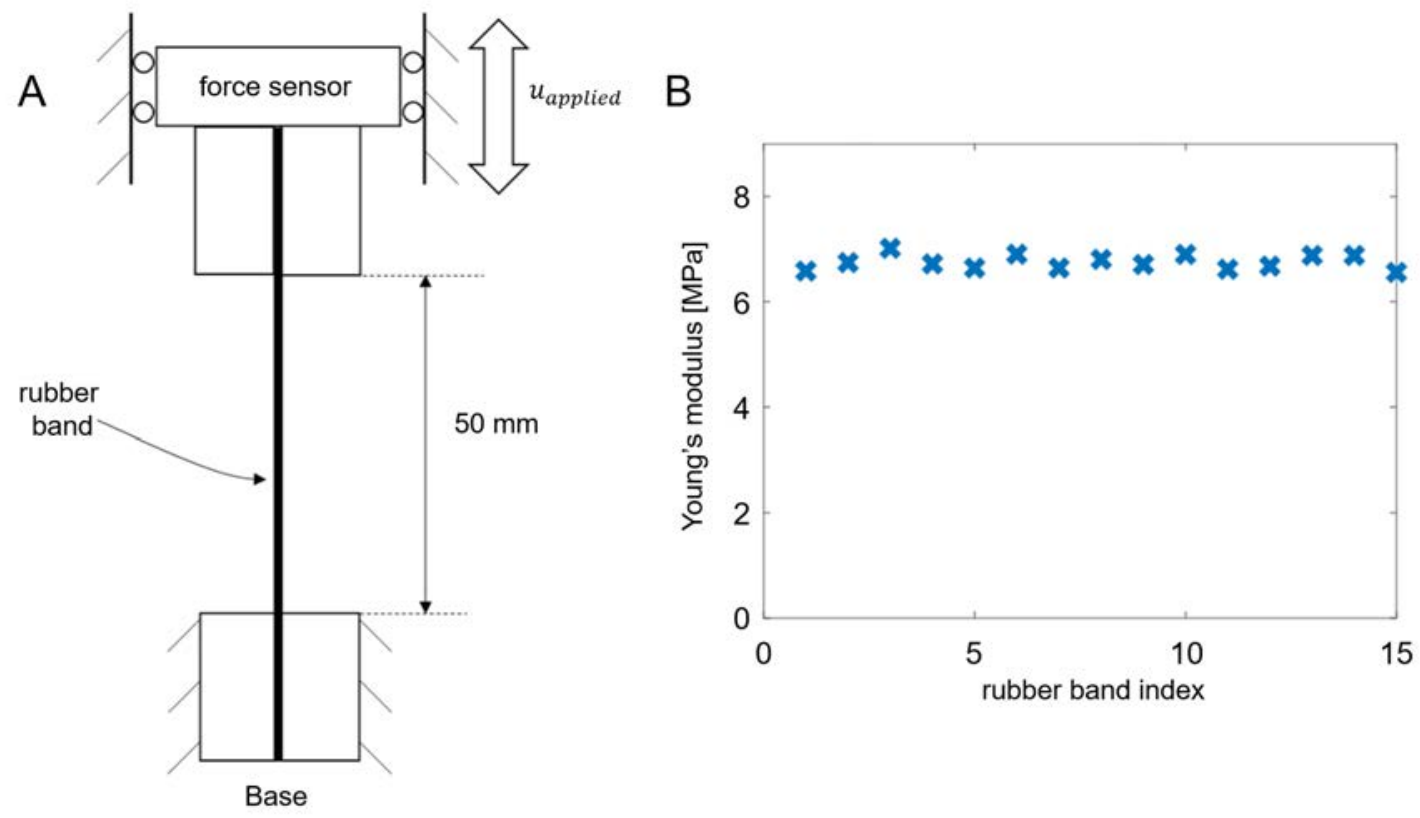

Figure S14: Characterization of the rubber bands. (A) Measurement setup for the calculation of the stiffness of rubber bands, (B) Calculated stiffness for 15 randomly selected bands.

Spring stiffness $k_{s}$ and $k_{l}$. To determine the stiffness $k_{s}$, we build a small structure consisting of four parallel hinges connected at both ends to interlocked LEGO plates (see Figure S15A) [1]. We start by fixing one column of LEGO plates and applying a displacement $u_{1}$ to the other one in direction perpendicular to the hinges (see Figure S15A), so that the hinges are only subjected to shearing deformations. The stiffness $k_{s}$ is then obtained from the measured force $F_{1}$ as

$$
k_{s}=\frac{F_{1}}{4 u_{1}}
$$

In Figure S15B we report the values of $k_{s}$ as a function of $l_{h}^{\text {inter }}$. Note that in the plot we compare the experimental results (green circular markers) to those obtained running FE simulations (star markers) and predicted analytically using [2]

$$
k_{s}=\frac{4 E I}{\left(l_{h}^{\text {inter }}\right)^{3}}, \quad \text { with } \quad I=\frac{w_{h}^{\text {inter }}\left(\delta_{h}^{\text {inter }}\right)^{3}}{12},
$$


where $E=4.33 \mathrm{GPA}, w_{h}^{\text {inter }}, \delta_{h}^{\text {inter }}, l_{h}^{\text {inter }}$ are the Young's modulus, width, thickness and length of the hinges, respectively. Given the very good agreement between the three sets of data, in our numerical analysis we use Eq. (S36) to obtain value of $k_{s}$ for given shim dimensions.

As for $k_{l}$, since it doesn't have a strong influence on the response of the system, we use a previously reported values $k_{l}=72 \mathrm{~N} / \mathrm{mm}[1]$.

A

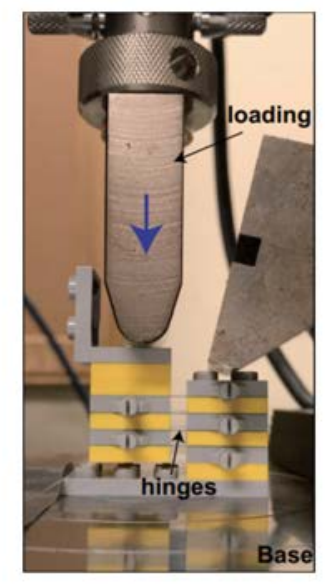

B

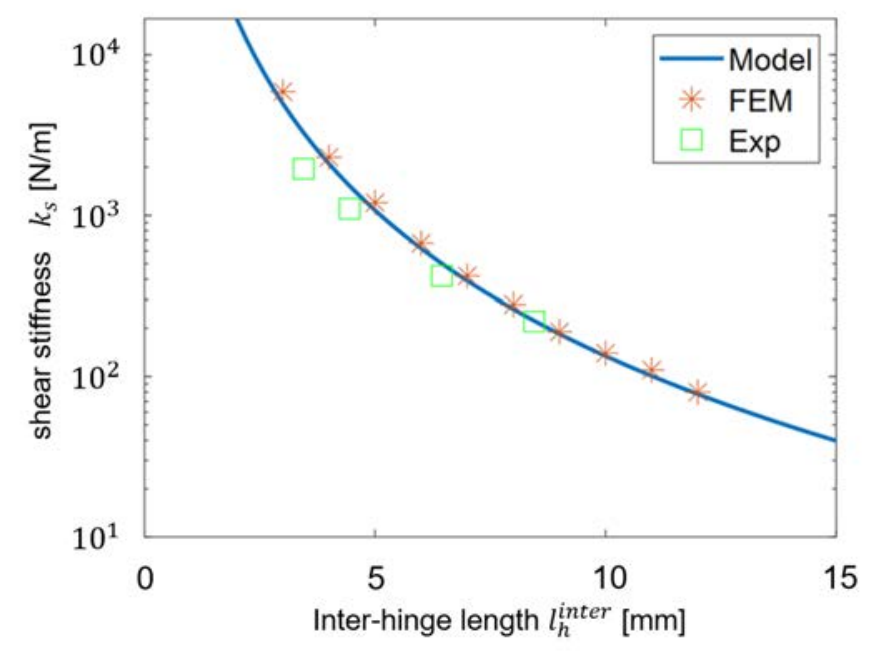

Figure S15: Characterization of $k_{s}$. (A) Measurement setup for the calculation of the shear stiffness $k_{s}$ of the inter-hinges, (B) Calculated shear stiffness based on the FEM, finite element and experimental measurements.

Friction coefficient $\mu_{\text {pin }}$. To measure the friction coefficient between the pins and the ground, $\mu_{\text {pin }}$, we mount the pins on a plate of known mass, $m$. Then, we place the plate with the pin over the surface used to conduct all our tests and connect the plate to a linear stage via a nylon thread. Finally, we use a load cell to measure the tension in the thread, $F_{s}$ when a displacement is imposed. The friction coefficient is estimated from the recorded data as (see Figure S16)

$$
\mu=\frac{F_{s}}{m g}
$$


where $g=9.8 \mathrm{~m} / \mathrm{s}^{2}$. In our simulations we then use

$$
\mu_{\text {pin }}=\max \mu=0.15 \text {. }
$$
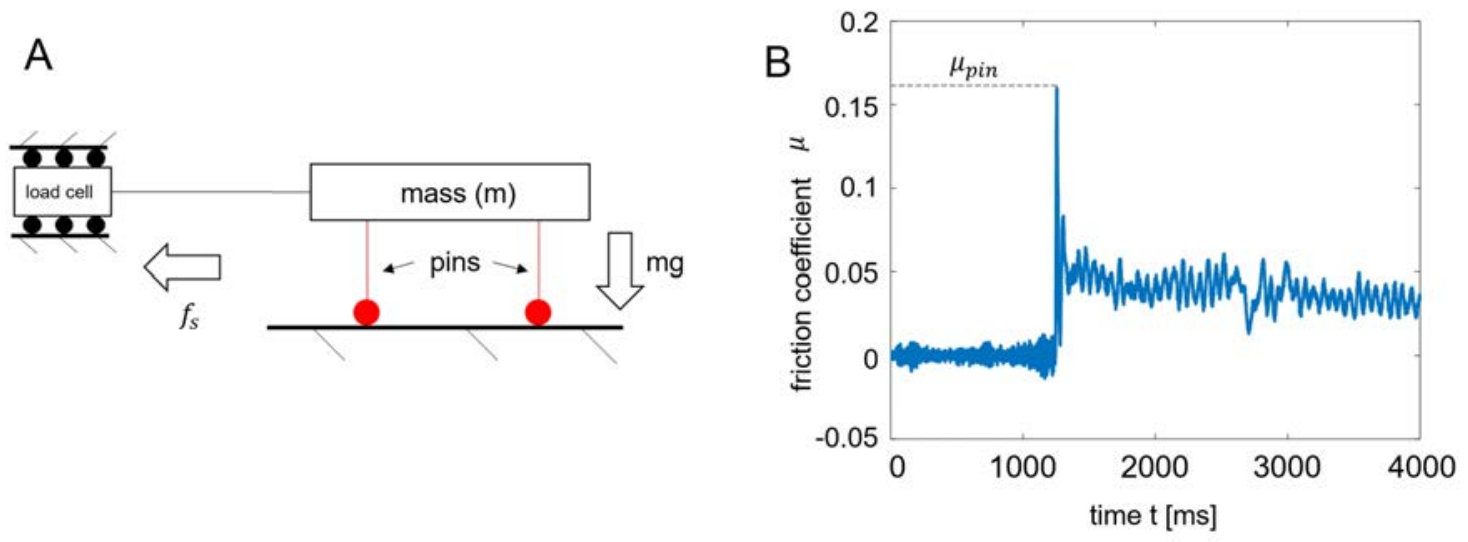

Figure S16: Characterization of pin friction. (A) Measurement setup for the calculation of the pin friction coefficient with the underlying substrate, (B) Calculated pin friction coefficient.

Friction coefficient $\mu_{s}$. To extract the sliding friction coefficient for the wheels, we used a rheometer (model HR-3- TA instruments). We glued the wheel to the rheometer lower plate at a distance $d_{\mathrm{rh}}$ from the rotation axis and attached to the rotating plate of the rheometer a disc of the same material used as substrate in our experiments (see Figure S17A). In our tests we controlled the rotation velocity of the upper disc, $\omega_{\text {rh }}$, and the applied normal force, $N_{\text {rh }}$, and measured the torque $T_{\mathrm{rh}}$. The friction coefficient of the wheels during sliding (the direction perpendicular to the rolling direction) $\mu_{s}$ is then evaluated as

$$
\mu_{s}=\frac{M_{\mathrm{rh}}}{d_{\mathrm{rh}} N_{\mathrm{rh}}} .
$$

In Figure S17B we show the evolution of $\mu_{s}$ as a function of the velocity of contact, $v_{s}=d_{\mathrm{rh}} \omega_{\mathrm{rh}}$ and find that the experimental data (marker) can be captured using (dashed line)

$$
\mu_{s}=0.95+7.8 v_{s}
$$


A

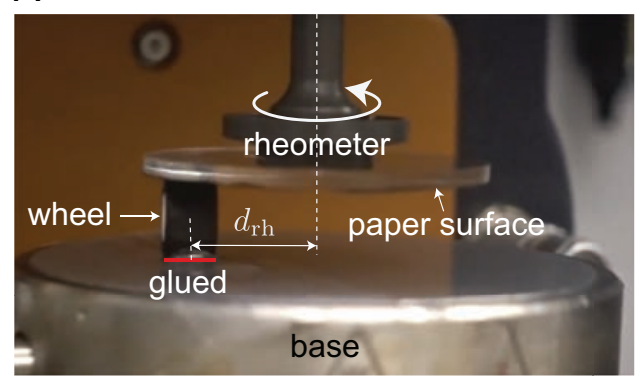

B

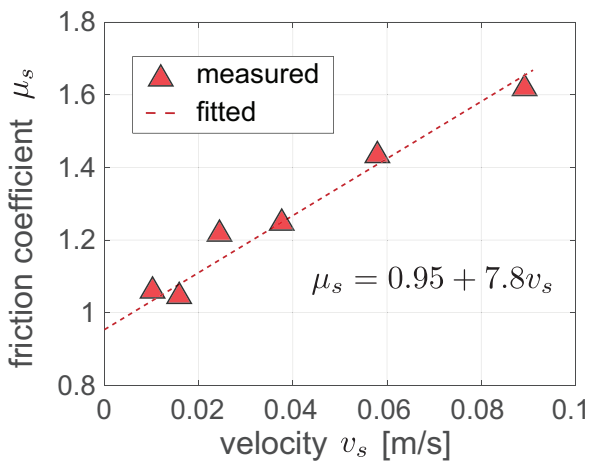

Figure S17: Characterization of wheel sliding friction. (A) Experimental setup to measure the wheel sliding friction coefficient $\mu_{s}$, (B) Measured sliding friction coefficient $\mu_{s}$ as a function of velocity $v_{s}$. 


\section{S3.4 Additional numerical results}
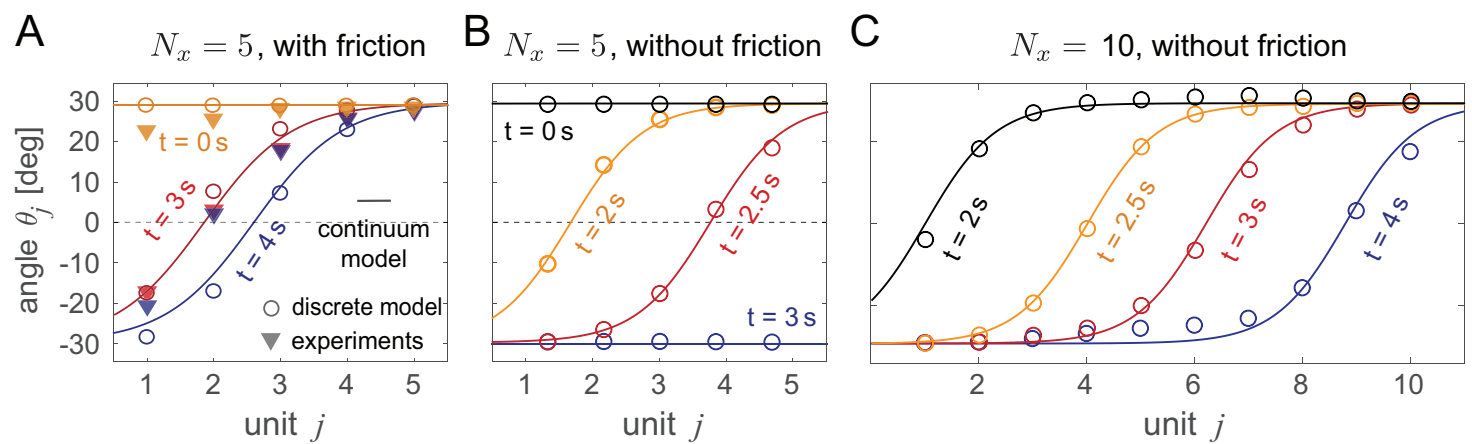

Figure S18: Effect of dissipation on wave propagation. Rotation $\theta_{j}$ of all units at different times $t$ as predicted by the discrete model (circular markers) and continuum model (lines) for a chain with $l_{h}^{\text {inter }}=8 \mathrm{~mm}$ and (A) $N_{x}=5$ in the presence of friction, (B) $N_{x}=5$ in the absence of friction and (C) $N_{x}=10$ in the absence friction. Note that the reported results for the continuum model are obtained by adjusting $\xi_{0}$ in Eq. (10) of the main text to best match the corresponding results for the discrete model. 

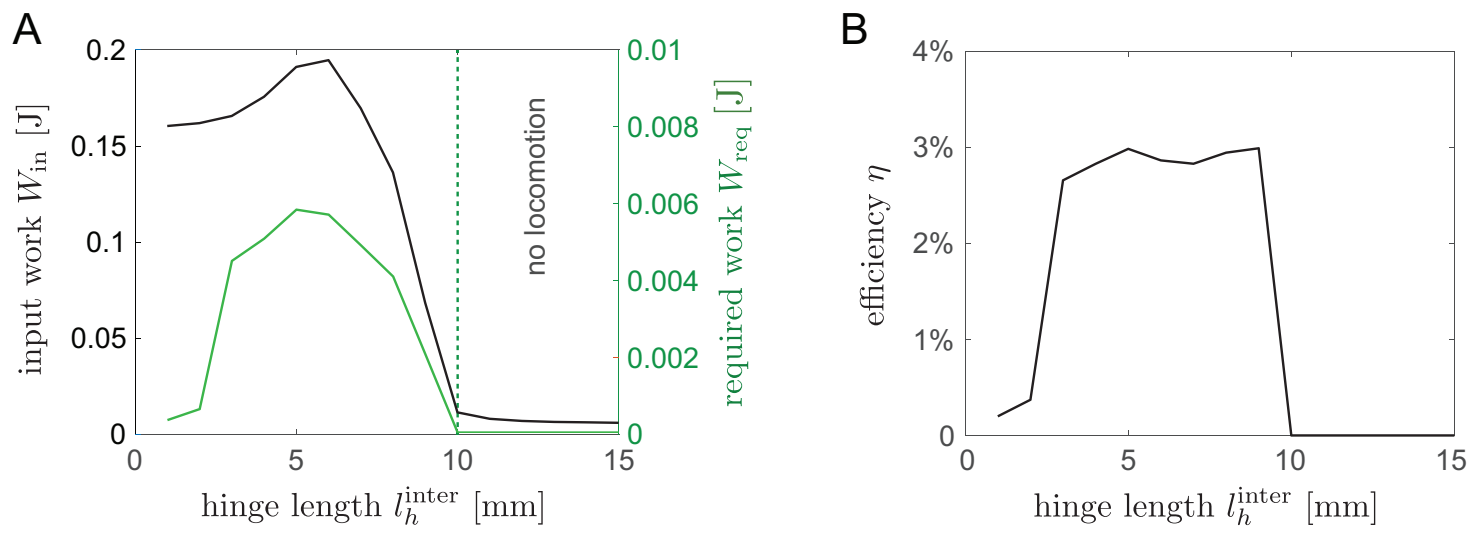

Figure S19: Efficiency of the proposed locomotion strategy. In order to evaluate the efficiency of the proposed locomotion strategy, we first calculated the work supplied to initiate the pulse

$$
W_{\text {in }}=\int_{0}^{t_{\text {cycle }}} M_{\text {act }}(t) \theta_{\text {act }}(t) d t .
$$

where $M_{\text {act }}(t)$ denotes the moment applied by the actuator to the unit cell to which is attached, $\theta_{\text {act }}(t)$ represents the the rotation of the crosses forming the unit cell to which the actuator is attached, and $t_{\text {cycle }}$ is the time period of one cycle. In (A) we present the evolution of $W_{\text {in }}$ as a function of $l_{h}^{\text {inter }}$ for a chain with $N_{x}=3$ (see black line). We find that $W_{\text {in }} \sim 0.15-0.2 \mathrm{~J}$ for $l_{h}^{\text {inter }}<6 \mathrm{~mm}$, with most of the work done to overcome the frictional forces between the wheels and the substrate as the unit cells switch between their two stable states. Then, $W_{\text {in }}$ rapidly decreases and almost vanishes for $l_{h}^{\text {inter }} \geq 10 \mathrm{~mm}$, since for long connecting shims the pulse does not propagate through the chain and only the unit to with the actuator is attached switches to the other stable configuration. Next, we calculated the work required for the chain to move on the substrate against friction as,

$$
W_{\text {req }}=m_{\text {tot }} g \mu_{s} \sin \theta_{s t} d_{\text {step }},
$$

where $m_{\mathrm{tot}}=129.5 \mathrm{~g}$ is the total mass of the chain, $\mu_{s}=0.95$ is the friction coefficient between the wheels and the substrate and $\theta_{s t}=29.4^{\circ}$ is the angle of the unit cells in their two stable phases. In agreement with the results for $W_{\text {in }}$ we find that for $l_{h}^{\text {inter }} \geq 10 \mathrm{~mm}$ the pulses do not have enough energy to propagate through the chain, so that both $d_{\text {step }}$ and $W_{\text {req }} \rightarrow 0$ (see green line in (A)). Finally, we calculated the efficiency of observed pulse-driven locomotion as

$$
\eta=\frac{W_{\text {req }}}{W_{\text {in }}}
$$

In (B) we report the evolution of $\eta$ as a function of $l_{h}^{\text {inter }}$ for a chain with $N_{x}=3$. We find that for $l_{h}^{\text {inter }}<3 \mathrm{~mm}$ and $l_{h}^{\text {inter }} \geq 10 \mathrm{~mm} \eta \rightarrow 0$, while for $3 \leq l_{h}^{\text {inter }}<10 \mathrm{~mm} \eta \approx 3 \%$. Although low, we note that such efficiency is comparable to that reported for the locomotion of soft robots [3]. 

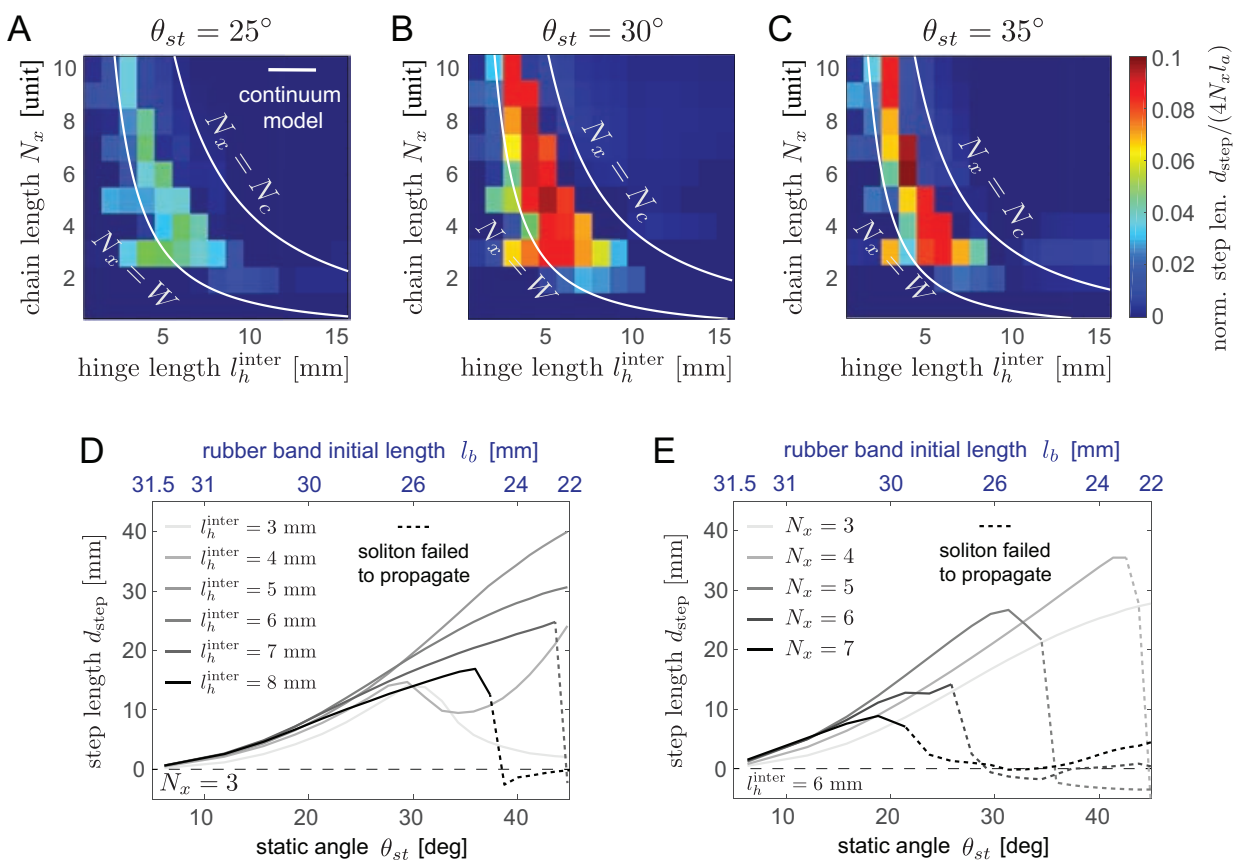

Figure S20: Numerical parametric studies on different geometrical parameters. (A)-(C) Effect of $N_{x}$ and $l_{h}^{\text {inter }}$ on $d_{\text {step }}$ for chains comprising units with (A) $\theta_{s t}=25^{\circ}$, (B) $\theta_{s t}=30^{\circ}$ and (C) $\theta_{s t}=35^{\circ}$. The white solid lines indicate the analytically predicted combinations of parameters resulting in $N_{x}=W$ and $N_{x}=N_{c}$. Note that the plot for $\theta_{s t}=30^{\circ}$ is also reported in the main text (Fig. 3F). (D)-(E) Effect of $\theta_{s t}$ (which is determined by the rubber band's initial length, $l_{b}$ ) on $d_{\text {step }}$ for chains with (D) $N_{x}=3$ and $l_{h}^{\text {inter }} \in[3,8] \mathrm{mm}$ and (E) $l_{h}^{\text {inter }}=6 \mathrm{~mm}$ and $N_{x} \in[3,7]$. The dashed lines indicate configurations for which the topological solitons fail to propagate to the end of the chain. 

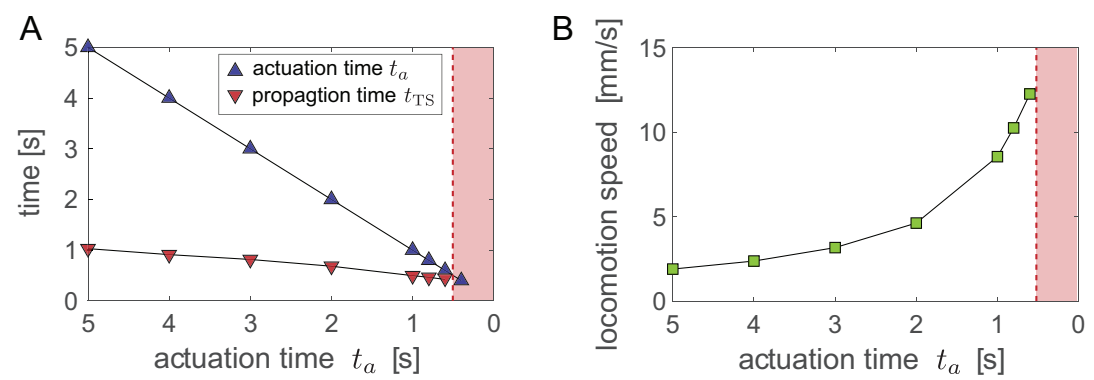

Figure S21: Effect of actuation time on the locomotion. Consider a chain with $N_{x}=3$ and $l_{h}^{\text {inter }}=6$ mm. (A) Effect of $t_{a}$ (i.e. the time that it takes the actuator to transition the unit to which it is attached from one stable phase to the other) to time it takes for the topological soliton to propagate through the chain, $t_{\mathrm{TS}}$. For $t_{a}<0.5 \mathrm{~s}, t_{\mathrm{TS}}<t_{a}$ and the topological solitons are not able to reach the end of the structure. (B) Evolution of the locomotion speed, $d_{\text {step }} /\left(2 t_{a}\right)$, as a function of the actuation time, $t_{a}$. By shortening the actuation time the crawling speed of the robot can be increased. However, such actuation time cannot be decreased indefinitely, since eventually it approaches the time that it takes the pulse to propagate through the chain. This is the limit condition at which the topological solitons are unable to reach the end of the structure before the opposite phase is triggered by the actuator, hindering the locomotion mechanism. 

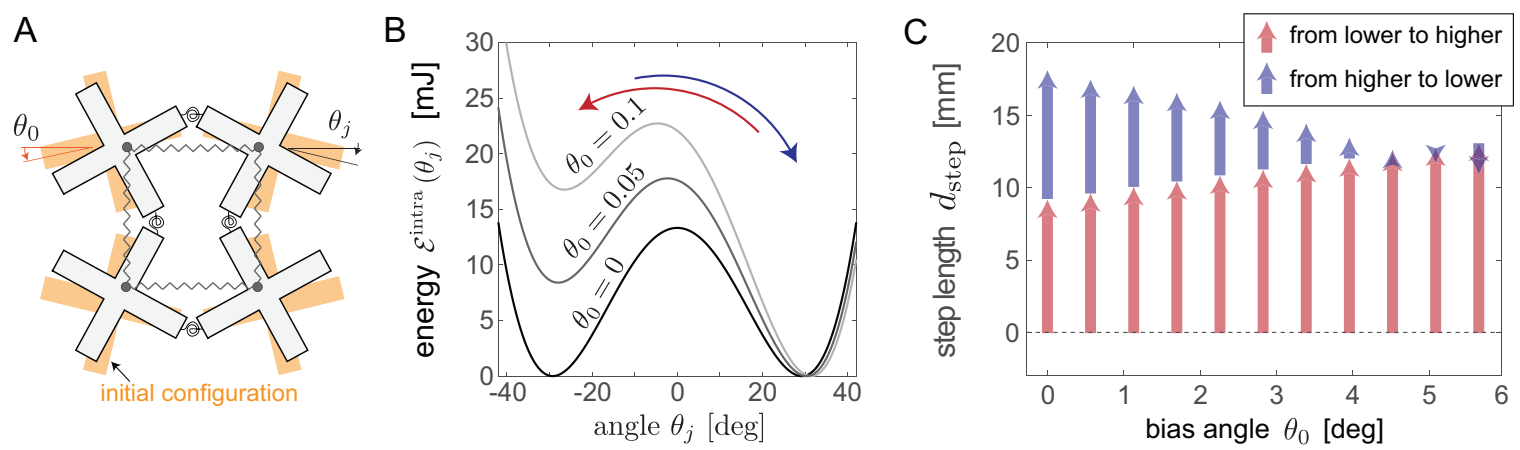

Figure S22: Effect of asymmetric energy landscape on the locomotion. (A) Schematics of a unit cell in which the crosses are rotated by an angle $\theta_{0}$ with respect to the horizontal and vertical direction before mounting the rubber band. The yellow crosses indicate their initial configuration before mounting the rubber band. The potential energy of this asymmetric unit can be written as,

$$
\mathcal{E}^{\text {intra }}\left(\theta_{j}\right)=2 k_{b}\left(l_{p} \cos \theta_{j}-l_{b}\right)^{2}+8 k_{\theta}\left(\theta_{j}-\theta_{0}\right)^{2},
$$

where $\theta_{j}$ is the internal angle, $k_{b}$ and $l_{b}$ are the stiffness and initial length of the rubber bands, $l_{p}$ is the maximal distance between the two posts and $k_{\theta}$ is the torsional stiffness of the hinges. To predict the dynamic response of a metamaterial comprising asymmetric units we introduce Eq. (S48) into Eq. (S10). (B) Elastic energy of the bistable unit, $\mathcal{E}_{\text {intra }}$, as predicted by the discrete model for unit cells with $\theta_{0}=0,0.05,0.1$. While for $\theta_{0}=0$ the energy profile of the unit cell possesses two minima of identical height, for $\theta_{0}>0$ the two energy minima become different. (C) Locomotion of a wheeled chain with $N_{x}=3, l_{h}^{\text {inter }}=6$ and unit cells with non-symmetric energy profile. The distance by the system moves when the units switch from the lower energy minima to the higher one (red arrows) is larger than that traveled when the units transition from the higher energy minima to the lower one (blue arrows). 

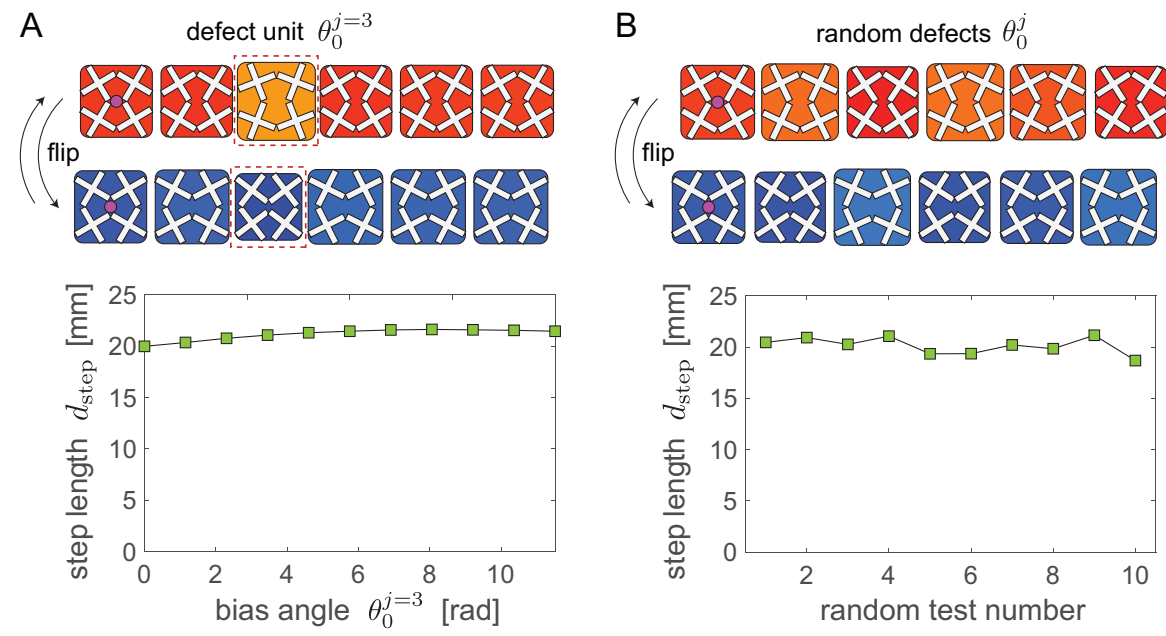

Figure S23: Effect of imperfections on the locomotion. Consider a of a chain with $N_{x}=6$ with $l_{h}^{\text {inter }}=4 \mathrm{~mm}$. (A) We assume only the $3 \mathrm{rd}$ unit in the chain to have an asymmetric energy profile generated by a bias angle $\theta_{0}$. We find that for $\theta_{0}$ up to $12^{\circ} d_{\text {step }}$ is minimally affected. (B) We assign a random bias angle $\theta_{0}^{j} \in\left[-6^{\circ}, 6^{\circ}\right]$ to all 6 units. Also in this case find consistent step length. 


\section{S3.5 Continuum model}

To get a deeper insight into the propagation of transition waves in our 1D chains, we simplify Eqs. (S22) to derive analytical solutions. Since here we consider a 1D structure, each unit has only two degrees of freedom: the translational displacement and internal rotation. For the sake of simplicity, we use $u_{j}$ and $\theta_{j}$ instead of $u^{[i, j]}$ and $\theta^{[i, j]}$ to indicate them. Under these assumptions, Eq. (S10) simplifies to

$$
\begin{aligned}
\mathcal{E}_{\text {ela }} & =\sum_{j=1}^{N_{x}} \mathcal{E}^{\text {intra }}\left(\theta_{j}\right)+\sum_{j=1}^{N_{x}-1}\left\{k_{s} l_{a}^{2}\left(\sin \theta_{j+1}-\sin \theta_{j}\right)^{2}\right. \\
& \left.+k_{l}\left[u_{j+1}-u_{j}+2 l_{a}\left(2 \cos \theta_{s t}-\cos \theta_{j+1}-\cos \theta_{j}\right)\right]^{2}\right\},
\end{aligned}
$$

and Eqs. (S22) simplify to

$$
\begin{aligned}
m \ddot{u}_{j}= & 2 k_{l}\left[u_{j+1}-2 u_{j}+u_{j-1}-2 l_{a}\left(\cos \theta_{j+1}-\cos \theta_{j-1}\right)\right] \\
J_{\theta} \ddot{\theta}_{j}= & 4 k_{b} l_{p} \sin \theta_{j}\left(l_{p} \cos \theta_{j}-l_{b}\right)-16 k_{\theta} \theta_{j}+2 k_{s} l_{a}^{2} \cos \theta_{j}\left(\sin \theta_{j+1}+\sin \theta_{j-1}-2 \sin \theta_{j}\right) \\
& -2 k_{l} l_{a} \sin \theta_{j}\left(u_{j+1}-u_{j-1}\right)
\end{aligned}
$$

Next, we introduce the continuous function $\theta(\xi, t)$ that interpolate the internal angle of the $j$-th unit as [4]

$$
\theta(\xi=j, t)=\theta_{j}(t), \text { and } u(\xi=j, t)=u_{j}(t)
$$

where $\xi$ denotes the unit number. Assuming that the width of the propagating waves is much larger than the unit cell size, the values of the neighbouring internal angles can be expressed using Taylor expansion as

$$
\begin{aligned}
& \theta(\xi=j+p, t)=\left[\theta+p \frac{\partial \theta}{\partial \xi}+\frac{1}{2} \frac{\partial^{2} \theta}{\partial \xi^{2}}\right]_{\xi=j} \\
& u(\xi=j+p, t)=\left[u+p \frac{\partial u}{\partial \xi}+\frac{1}{2} \frac{\partial^{2} u}{\partial \xi^{2}}\right]_{\xi=j}
\end{aligned}
$$

where $p, q \in\{-1,1\}$. Further, we approximate the trigonometric functions as

$$
\sin \theta \approx \theta-\frac{\theta^{3}}{6}, \text { and } \cos \theta \approx 1-\frac{\theta^{2}}{2}
$$


Substitution of Eqs. (S51) - (S53) into Eqs. (S50) yields

$$
\begin{aligned}
& m \frac{\partial^{2} u}{\partial t^{2}}=2 k_{l} \frac{\partial^{2} u}{\partial \xi^{2}}+8 k_{l} l_{a} \theta \frac{\partial \theta}{\partial \xi}, \\
& J_{\theta} \frac{\partial^{2} \theta}{\partial t^{2}}=2 k_{s} l_{a}^{2} \frac{\partial^{2} \theta}{\partial \xi^{2}}-\left(2 k_{b} l_{p}^{2}+4 k_{l} l_{a}^{2}\right)\left(\theta^{3}-\theta_{s t}^{2} \theta\right)-4 k_{l} l_{a} \theta \frac{\partial u}{\partial \xi},
\end{aligned}
$$

To analytically solve Eq. (S54), we introduce the traveling coordinate $\zeta=\xi-c t$ ( $c$ denting the propagating velocity of the transition wave) and rewrite Eq. (S54) as

$$
\begin{aligned}
\frac{\partial^{2} u}{\partial \zeta^{2}} & =-\frac{8 k_{l} l_{a} \theta \frac{\partial \theta}{\partial \zeta}}{2 k_{l}-m c^{2}}, \\
\frac{\partial^{2} \theta}{\partial \zeta^{2}} & =\frac{\left(2 k_{b} l_{p}^{2}+8 k_{l} l_{a}^{2}\right)\left(\theta^{3}-\theta_{s t}^{2} \theta\right)+4 k_{l} l_{a} \theta \frac{\partial u}{\partial \zeta}}{2 k_{s} l_{a}^{2}-c^{2} J_{\theta}} .
\end{aligned}
$$

We then integrate Eq. (S55a) to obtain

$$
\frac{\partial u}{\partial \zeta}=-\frac{4 k_{l} l_{a} \theta^{2}}{2 k_{l}-m c^{2}}+C
$$

where $C$ is an integration constant. By imposing that

$$
\partial u / \partial \zeta=0 \text { and } \theta=\theta_{s t} \text { as } \zeta \rightarrow \infty
$$

we find that

$$
C=\frac{4 k_{l} l_{a} \theta_{s t}^{2}}{2 k_{l}-m c^{2}}
$$

and Eq. (S56) can be written as

$$
\frac{\partial u}{\partial \zeta}=\frac{4 k_{l} l_{a}\left(\theta_{s t}^{2}-\theta^{2}\right)}{2 k_{l}-m c^{2}}
$$

Substitution of Eq. (S59) into Eqs. (S54b) yields

$$
\frac{\partial^{2}}{\partial \zeta^{2}} \theta=\frac{k_{b} l_{p}^{2}-\frac{4 k_{l} l_{a}^{2} c^{2} / c_{u}^{2}}{1-c^{2} / c_{u}^{2}}}{k_{s} l_{a}^{2}\left(1-c^{2} / c_{\theta}^{2}\right)} \theta\left(\theta-\theta_{s t}\right)\left(\theta+\theta_{s t}\right)
$$

where $c_{\theta}=\sqrt{2 k_{s} l_{a}^{2} / J_{\theta}} \approx 311$ unit/s and $c_{u} \approx 1788$ unit/s. Since the pulse speed observed in our experiments is $c \approx 5$ unit/s, we can assume that $c / c_{u} \approx 0$ and $c / c_{\theta} \approx 0$ and Eq. (S60) 
simplifies to

$$
\frac{\partial^{2}}{\partial \zeta^{2}} \theta=\frac{k_{b} l_{p}^{2}}{k_{s} l_{a}^{2}} \theta\left(\theta-\theta_{s t}\right)\left(\theta+\theta_{s t}\right)
$$

Eq. (S61) is a Klein-Gordon Equation with quadratic and cubic nonlinearities and admits a kink solution with form

$$
\theta= \pm \theta_{s t} \tanh \left(\frac{\xi-c\left(t-t_{0}\right)}{W}\right)
$$

where $t_{0}$ is a time shift and $W$ denotes the width of the pulse. By substituting the solution (Eq. S62) into Eq. (S61), we find that the latter is identically satisfied only if

$$
W=\frac{l_{a}}{\theta_{s t} l_{p}} \sqrt{\frac{2 k_{s}}{k_{b}}}=\sqrt{\frac{k_{s} l_{a}^{2} \theta_{s t}^{2}}{\Delta \mathcal{E}^{\text {intra }}}} .
$$

Eq. (S63) indicates that $W$ is determined by the balance between the energy associated to the interaction between units $\left(\sim k_{s} l_{a}^{2} \theta_{s t}^{2}\right)$ and the local energy barrier $\left(\Delta \mathcal{E}^{\text {intra }}\right)$. Consistently with our experimental observations, Eq. (S63) indicates that a chain with strong interaction energy (i.e., short/stiff inter-hinges) supports wider transition waves, whereas a chain with weak interaction energy (i.e., long/flexible inter-hinges) supports thinner transition waves.

Finally, by substituting Eq. (S62) into Eq. (S59) and integrating the latter with respect to $\zeta$ the translational component of the wave can be obtained as

$$
u=2 l_{a} W \theta_{s t}^{2} \tanh \left(\frac{\xi-c\left(t-t_{0}\right)}{W}\right) .
$$

Next, we use the derived analytical solution to predict $N_{c}$ (i.e. the maximum number of units resulting in a chain through which the propagating pulses can reach the opposite end). Towards this end, we introduce Eqs. (S52) and (S53) into Eq. (S49) and account for Eq. (S59) to write the elastic energy of the chain in a continuum sense as,

$$
\mathcal{E}_{\mathrm{ela}} \approx \int_{-\infty}^{\infty}\left[\mathcal{E}^{\text {intra }}(\theta)+k_{s} l_{a}^{2}\left(\frac{\partial \theta}{\partial X}\right)^{2}\right] d X .
$$

The energy associated to a topological soliton can then be estimated by subtracting the elastic energy of a chain that deforms homogeneously (for which $\theta= \pm \theta_{s t}$ ) from that of a chain with 
a topological soliton (for which $\theta$ is defined by Eq. (S62)),

$$
\begin{aligned}
\mathcal{E}_{\text {ts }} & =\left.\mathcal{E}_{\text {ela }}\right|_{\theta=\theta(\zeta)}-\left.\mathcal{E}_{\text {ela }}\right|_{\theta=\theta_{s t}} \\
& =\int_{-\infty}^{\infty}\left\{\mathcal{E}_{\text {intra }}(\theta(\zeta))+2 k_{s} l_{a}^{2}\left[\frac{\partial \theta(\zeta)}{\partial \zeta}\right]^{2}-\mathcal{E}_{\text {intra }}\left(\theta_{s t}\right)\right\} d \zeta \\
& =\frac{4}{3} k_{b} l_{p}^{2} \theta_{s t}^{4} W+\frac{4 k_{s} l_{a}^{2} \theta_{s t}^{2}}{3 W} \\
& =2 \theta_{s t}^{3} l_{a} l_{p} \sqrt{2 k_{s} k_{b}}
\end{aligned}
$$

Note that when calculating $\mathcal{E}_{t s}$ we neglected the kinetic energy of the topological solitons, since the pulses propagate slowly. Finally, we calculate $N_{c}$ by comparing $\mathcal{E}_{\text {ts }}$ to the energy required to switch a unit cell from one stable state to the other

$$
N_{c}=\frac{\mathcal{E}_{\mathrm{ts}}}{\Delta \mathcal{E}^{\text {intra }}}=\frac{4 l_{a}}{\theta_{s t} l_{p}} \sqrt{\frac{2 k_{s}}{k_{b}}}=4 W .
$$

For chain with $N_{x}>N_{c}$ we expect a soliton excited at one end of the chain to remain trapped inside the chain, whereas for a chain with $N_{x}<N_{c}$ we expect the topological soliton to reach the opposite end switching all units. 


\section{S4 Description of Supporting Movies}

\section{Movie S1: Bistable unit cell.}

The building block of our mechanical metamaterial is a bistable unit that comprises four rigid crosses connected by elastic hinges and a pre-stretched elastic band. The static response of our unit is characterized using a uniaxial testing apparatus (Instron - 5960 series) and a set of levers and rigid joints that convert the applied linear displacement into rotation of the crosses.

\section{Movie S2: Topological solitons in chains.}

Propagation of topological solitons in chains with $\left(l_{h}^{\text {inter }}, N_{x}\right)=(4 \mathrm{~mm}, 5),(6 \mathrm{~mm}, 5),(8$ $\mathrm{mm}, 5)$ and $(8 \mathrm{~mm}, 4)$. Note that $l_{h}^{\text {inter }}$ denotes the length of that hinges that connect neighboring bistable units and $N_{x}$ represents the number of unit cells in the chain.

\section{Movie S3: Friction modification via wheels.}

To tune the frictional forces, we add to each cross in the metamaterial a pair of wheels. Here we show that for $\theta_{j}=0^{\circ}$ the unit cell starts to move at a tilting angle of $\sim 6^{\circ}$. Differently, for start $\theta_{j}=30^{\circ}$ the unit cell does not move up to a tilting angle of $25^{\circ}$, indicating that the frictional force is highly affected by $\theta_{j}$.

\section{Movie S4: Locomotion via topological solitons.}

(i) Effect of $N_{x}$ and $l_{h}^{\text {inter }}$ on the locomotion of wheeled chains with $\left(l_{h}^{\text {inter }}, N_{x}\right)=(4 \mathrm{~mm}$, $3),(6 \mathrm{~mm}, 3),(8 \mathrm{~mm}, 3),(15 \mathrm{~mm}, 3)$ and $(6 \mathrm{~mm}, 6)$. Note that $l_{h}^{\text {inter }}$ denotes the length of that hinges that connect neighboring bistable units and $N_{x}$ represents the number of unit cells in the chain.

(ii) Effect of the actuation time $t_{a}$ on the locomotion of a wheeled chain with $l_{h}^{\text {inter }}=6 \mathrm{~mm}$ and $N_{x}=3$. As the $t_{a}$ becomes shorter, the topological solitons fail to reach the end of the chain.

(iii) Effect of defects on the locomotion of a wheeled chain with $l_{h}^{\text {inter }}=4 \mathrm{~mm}$ and $N_{x}=6$. The defects minimally influence the locomotion of the chain. 


\section{Movie S5: Locomotion with multiple actuators.}

When connecting two actuators to the leftmost and rightmost unit, we can make a chain with $N_{x}=6$ and $l_{h}^{\text {inter }}=6 \mathrm{~mm}$ to crawl. Moreover, by tuning the normalized time shift between the two actuators, $\tau$, the chain can move both leftwards (for $\tau>0$ ) and rightwards (for $\tau>0$ ). Further for $\tau=0$ the chain does not move.

Finally, the second actuator not only enables us to control the direction of motion, but also improves the robustness of the system, as in case of a unit failure (i.e. a pair of shims are cut) the chain splits into two independent and functional systems.

\section{Movie S6: Propagation of waves in 2D systems.}

Propagation of waves in a 2D structure comprising $6 \times 6$ unit cells with $l_{h}^{\text {inter }}=3 \mathrm{~mm}$. We simulated the behavior of the structure when the actuator is placed at three different locations (indicated by a star marker).

\section{Movie S7: Locomotion of 2D structures.}

Locomotion of a 2D structure comprising $2 \times 2$ unit cells and an actuator connected to its top-left cell. We consider chains with $l_{h}^{\text {inter }}=5 \mathrm{~mm}, 7 \mathrm{~mm}$ and $15 \mathrm{~mm}$.

To better control the rotation of the 2D structure, we attach two actuators to the left two unit cells of the $2 \times 2$ metamaterial with $l_{h}^{\text {inter }}=11 \mathrm{~mm}$. The rotation direction of the structure can be effectively controlled by tuning the normalized time shift between the two actuators, $\tau$.

By simply varying $\tau$ we can guide the structure along complex paths comprising straight segments and turns. 


\section{References}

[1] Bolei Deng, Pai Wang, Qi He, Vincent Tournat, and Katia Bertoldi. Metamaterials with amplitude gaps for elastic solitons. Nature Communications, 9:3410, 2018.

[2] Shorya Awtar, Alexander H Slocum, and Edip Sevincer. Characteristics of beam-based flexure modules. ASME Jounral of Mechanical Design, 129, 2007.

[3] Langquan Shui, Liangliang Zhu, Zhe Yang, Yilun Liu, and Xi Chen. Energy efficiency of mobile soft robots. Soft Matter, 13(44):8223-8233, 2017.

[4] Bolei Deng, Jordan Raney, Vincent Tournat, and Katia Bertoldi. Elastic vector solitons in soft architected materials. Physical Review Letters, 118(20), 2017. 